\title{
Influence of spin-orbit interaction on the electrical conductivity of three-dimensional disordered systems
}

\author{
B. J. Spisak ${ }^{1}$, A. Paja ${ }^{*, 1}$, and G. J. Morgan ${ }^{2}$ \\ ${ }^{1}$ Faculty of Physics and Applied Computer Science, AGH University of Science and Technology, \\ Al. Mickiewicza 30, 30-059 Kraków, Poland \\ 2 Department of Physics and Astronomy, University of Leeds, Leeds LS2 9JT, United Kingdom
}

Received 30 July 2004, revised 3 January 2005, accepted 15 March 2005

Published online 4 May 2005

PACS 71.70.Ej, 72.10.-d, 72.80.Ng

A calculation of the effects of spin-orbit interaction on electron transport in 3D disordered systems is presented. We use the Wigner density matrix to construct self-consistent transport equations which can be used to discuss weak localization (quantum interference) effects and even strong localization. The formula for the transport relaxation time has been obtained. The change of the resistivity due to spin-orbit interaction is negative in agreement with experiment.

(C) 2005 WILEY-VCH Verlag GmbH \& Co. KGaA, Weinheim

\section{Introduction}

The electronic transport properties of disordered or complex conductors have been of great interest both theoretically and experimentally over the past years [1]. The rapid development of spin electronics, which has taken place in recent years [2], reinforces the need for a good understanding of the effects of spin-orbit interaction [3, 4]. The effects of the spin-orbit interaction have wider consequences in the context of electron transport in magnetic tunnel junctions [5] and other nanostructures [6-9]. It is particularly important in disordered materials where quantum interference (QI) gives rise to weak localization (WL) and, on the other hand, various factors can destroy this subtle phenomenon [10, 11]. We consider the spin-orbit interaction when $\boldsymbol{B}=\mathbf{0}$ in this paper, which is complex matter in itself, as a prelude to the case when $\boldsymbol{B} \neq \mathbf{0}$. A number of experiments [12-15] have shown that heavy atoms, such as e.g. $\mathrm{Au}$, give rise to substantial changes in the electrical resistivity of disordered systems due to the strong spin-orbit interaction. To explain the experimental results the authors of the quoted papers used to apply the theory of Fukuyama and Hoshino [16] which was an extension of a previous theory of Kawabata [17, 18] developed for the magnetoconductivity of disordered systems. However we have argued [19] that Kawabata's theory, originally formulated for degenerate semiconductors, is of uncertain validity for amorphous metals, where very often the Ioffe-Regel criterion is not valid [20] or one is working at the limit of its validity. In the discussion of electron transport properties we will consider nearly free electrons moving in a disordered system of weak potentials and neglect electron-electron scattering effects and internal fields arising from the electron-electron interaction. This is often the model used for disordered systems but real systems are usually much more complex, for example many amorphous alloys are composed of transition metals with conducting $d$-bands. In the analysis of experiments on such systems it is often hoped that theories for simple models have a universal character but there is no guarantee that this is true. For simplicity we will consider an amorphous system with just one type of atomic species describable by a pseudopotential and the total potential is $U_{0}(\boldsymbol{R})$. Of course the use of a pseudopotential

"Corresponding author: e-mail: paja@uci.agh.edu.pl, Phone: +48 1261725 56, Fax: +48 126341247 
in the context of spin-orbit interaction has some simplifying aspects in that the core parts of the pseudowaves are in fact most important in determining the size of the interaction as well as the derivative of the true potential. For our present purpose we will simply regard the magnitude of the interaction as an adjustable parameter $[21,22]$ which will be discussed at a later stage. The spin-orbit interaction is defined by [23]

$$
U_{\mathrm{SO}}(\boldsymbol{R})=a_{\mathrm{SO}} \hat{\sigma} \cdot\left[\nabla U_{0}(\boldsymbol{R}) \times \hat{\boldsymbol{p}}\right],
$$

where $\hat{\boldsymbol{p}}$ is the momentum operator, $\hat{\sigma}$ denotes the Pauli spin matrices. The extension to a more than one-component system is straightforward but further complicates an already elaborate theory.

The role of spin-orbit interaction increases rapidly with the atomic number [24] and therefore this effect was clearly observed many years ago in metals where electrons are scattered by heavy ions placed in a matrix of lighter ones [13] or on the surface of thin films [25]. This is important effect in the context of QI effects or ' $2 k_{F}$ '-scattering $[25,26]$ where multiple scattering of electrons inhibits the diffusion of electrons which leads to the alternative expression [27, 28], namely a weak localization corrections to the usual Boltzmann equation description of conduction processes [29]. This term is sometimes used because of its importance in the study of $2 \mathrm{D}$ semiconductors where the singular ' $2 k_{F}$ '-scattering can lead to an infinite resistance [30].

The motivation for this work is that we have some reservations about existing treatments [19] but we are building on the previous ideas of others in this paper and are seeking to establish the relationship between this and previous work.

In this paper we make use of previous theory [26] utilizing the Wigner density matrix [31-33] or distribution function to develop generalized transport equations which have a form similar to the linearized Boltzmann equation. This is strongly connected to one of the earliest approach to transport theory namely that of Kohn and Luttinger [34,35]. Many treatments of transport problems utilize the Kubo formula and Green function methods where the Boltzmann type of transport equations is replaced by Bethe-Salpeter equations [36, 37]. Both approaches have their merits but here we use the Wigner density matrix as we believe that it is more transparent for some purposes although there is of course a great similarity in the structure of the equations obtained. We would also like to underline that many authors have recently used the Wigner function formulation of the quantum theory of electron transport in various systems [38-43] which was proposed in [26] for the first time.

In Section 2 we describe the Wigner representation for the case when the electron spin indices are included and also the spin-orbit interaction. The conductivity (for a constant electric field $\boldsymbol{E}$ ) only depends on the average value of the density matrix and we introduce some reasonable simplifications of the general equations coupled by the spin-orbit interaction. In Section 3 we then further simplify matters by constructing a hierarchy of configurationally averaged Wigner equations which contain the important features which lead to ' $2 k_{F}$ '-scattering. These equations are of necessity extremely complicated, but they are important to the rest of the paper and have their analogues in other self-consistent Green function methods and the Bethe-Salpeter equation. In Section 4 we reduce the complicated generalized equations to forms which yield the salient effects of the spin-orbit interaction and show that this interaction gives rise to the decrease of electrical resistivity in the considered systems.

\section{Derivation of transport equation}

The linearized equation for the one-particle density matrix $\rho$ describing the deviation of a system from equilibrium in a constant electric field $\boldsymbol{E}$ is [34,35]

$$
\begin{aligned}
& i \hbar \varepsilon \rho_{s^{\prime} s}\left(\boldsymbol{k}^{\prime}, \boldsymbol{k}\right)-\sum_{\boldsymbol{k}^{\prime \prime}} \sum_{s^{\prime \prime}}\left\{H_{s^{\prime} s^{\prime \prime}}\left(\boldsymbol{k}^{\prime}, \boldsymbol{k}^{\prime \prime}\right) \rho_{s^{\prime \prime} s}\left(\boldsymbol{k}^{\prime \prime}, \boldsymbol{k}\right)-\rho_{s^{\prime} s^{\prime \prime}}\left(\boldsymbol{k}^{\prime}, \boldsymbol{k}^{\prime \prime}\right) H_{s^{\prime \prime} s^{\prime}}\left(\boldsymbol{k}^{\prime \prime}, \boldsymbol{k}^{\prime}\right)\right\} \\
& =i \hbar e \boldsymbol{E} \sum_{\boldsymbol{k}^{\prime \prime}} \sum_{s^{\prime \prime}}\left\{\boldsymbol{R}_{s^{\prime} s^{\prime \prime}}\left(\boldsymbol{k}^{\prime}, \boldsymbol{k}^{\prime \prime}\right) \rho_{s^{\prime \prime} s}^{0}\left(\boldsymbol{k}^{\prime \prime}, \boldsymbol{k}\right)-\rho_{s^{\prime} s^{\prime \prime}}^{0}\left(\boldsymbol{k}^{\prime}, \boldsymbol{k}^{\prime \prime}\right) \boldsymbol{R}_{s^{\prime \prime} s}\left(\boldsymbol{k}^{\prime \prime}, \boldsymbol{k}^{\prime}\right)\right\},
\end{aligned}
$$


where $\hat{H}$ is the Hamiltonian of the system, $\hat{\boldsymbol{R}}$ is a position operator in a plane wave basis and the $s$ indices denote the spin states. The electric field (or electrochemical potential gradient) cannot be exactly constant in an inhomogeneous medium but this simplification is often made and is not the issue in this work. The infinitesimal $\varepsilon$ results from switching on the electric field in an adiabatic manner. An appropriate approximate solution of Eq. (2) should ideally yield a description of the transport phenomena which are linear in the electric field, including weak localization and strong localization, as an alternative to the use of Green function methods and the Bethe-Salpeter equation [37]. It is our preference to use the Wigner representation of Eq. (2) [31-33] as we believe it is simpler to envisage some of the approximations made in an almost classical fashion. Consider an arbitrary one-particle operator $\hat{O}$ (which may be a product of two one-particle operators as in our case) in the momentum representation. The matrix elements of this operator in the Wigner representation are given by

$$
O_{W}(\boldsymbol{R}, \boldsymbol{K})=\frac{1}{\Omega} \sum_{q}\left\langle\boldsymbol{K}+\frac{1}{2} \boldsymbol{q}|\hat{O}| \boldsymbol{K}-\frac{1}{2} \boldsymbol{q}\right\rangle \mathrm{e}^{i q \cdot \boldsymbol{R}} .
$$

There are alternative definitions of the Wigner function [10] but (3) is sufficient for our purpose. The Wigner representation of the density operator is called the Wigner distribution function [23] and it is analogous to the classical probability of finding a particle at a position $\boldsymbol{R}$ with momentum $\hbar \boldsymbol{K}$. One of the important properties of the equilibrium Wigner distribution function which distinguishes it from the classical equilibrium distribution function is that it may be negative in any subregion of phase space although an average over the $\boldsymbol{K}$ variable yields a positive definite quantity. Of course a negative deviation from equilibrium does not produce quite the same dilemma. We will extend this method to describe the spin variables in the following way. Denoting the $z$-components of spin by $\alpha$ and $\beta$, we define the Wigner matrix as follows

$$
\rho_{s^{\prime} s}(\boldsymbol{R}, \boldsymbol{K})=\left[\begin{array}{cc}
\rho_{\alpha \alpha}(\boldsymbol{R}, \boldsymbol{K}) & \rho_{\alpha \beta}(\boldsymbol{R}, \boldsymbol{K}) \\
\rho_{\beta \alpha}(\boldsymbol{R}, \boldsymbol{K}) & \rho_{\beta \beta}(\boldsymbol{R}, \boldsymbol{K})
\end{array}\right] .
$$

From a classical point of view the quantity $\rho_{\alpha \alpha}(\boldsymbol{R}, \boldsymbol{K})$ determines the average number of electrons in the state $\boldsymbol{K}$ with spin $\alpha$ in the element of volume $d^{3} R$ at the position $\boldsymbol{R}$ and similarly $\rho_{\beta \beta}(\boldsymbol{R}, \boldsymbol{K})$. The off-diagonal elements describe the fluctuations from the average values.

Applying the same procedure to Eq. (2) gives

$$
\begin{aligned}
& {\left[\frac{\hbar \boldsymbol{K}}{m} \cdot \nabla+\varepsilon\right] \rho_{s^{\prime} s}(\boldsymbol{R}, \boldsymbol{K})+\frac{2}{\hbar} \sum_{q} \mathfrak{I}\left\{U_{0}(\boldsymbol{q}) \mathrm{e}^{i q \cdot \boldsymbol{R}}\right\} \rho_{s^{\prime} s}\left(\boldsymbol{R}, \boldsymbol{K}+\frac{1}{2} \boldsymbol{q}\right)} \\
& +i \frac{b_{\mathrm{SO}}}{2 \hbar} \sum_{s^{\prime \prime}} \sum_{q}\left[\hat{\Lambda}(\boldsymbol{q}, \boldsymbol{K}) \rho_{s^{\prime} s^{\prime \prime}}\left(\boldsymbol{R}, \boldsymbol{K}+\frac{1}{2} \boldsymbol{q}\right) \cdot \sigma_{s^{\prime \prime} s}-\hat{\Lambda}^{*}(\boldsymbol{q}, \boldsymbol{K}) \cdot \sigma_{s^{\prime} s^{\prime \prime}} \rho_{s^{\prime \prime} s}\left(\boldsymbol{R}, \boldsymbol{K}+\frac{1}{2} \boldsymbol{q}\right)\right] \\
& =-e \boldsymbol{E} \cdot \sum_{s^{\prime \prime}}\left[\frac{\hbar \boldsymbol{K}}{m} \delta_{s^{\prime} s^{\prime \prime}}+\boldsymbol{v}_{s^{\prime} s^{\prime \prime}}^{\mathrm{SO}}(\boldsymbol{K})\right] \phi_{s^{\prime \prime} s}(\boldsymbol{R}, \boldsymbol{K}),
\end{aligned}
$$

where $\boldsymbol{v}_{s^{\prime} s}^{\text {SO }}(\boldsymbol{K})$ results from the commutator of the spin-orbit interaction with the position variable, $b_{\mathrm{SO}}=\hbar a_{\mathrm{SO}}, \phi(\boldsymbol{R}, \boldsymbol{K})$ is the Wigner representation of the derivative of the Fermi-Dirac function, and $\hat{\Lambda}(\boldsymbol{q}, \boldsymbol{K})$ is defined by the formula

$$
\hat{\Lambda}(\boldsymbol{q}, \boldsymbol{K})=U_{0}(\boldsymbol{q}) \mathrm{e}^{i \boldsymbol{q} \cdot \boldsymbol{R}}[\boldsymbol{q} \times(\nabla-2 i \boldsymbol{K})],
$$

where $U_{0}(\boldsymbol{q})$ is the Fourier transform of the static potential in the solid. At this point it should again be noted that many calculations of the properties of metals with nearly free electron like densities of state are carried out using pseudopotentials which replace the deep atomic-like potentials and enable low order perturbation theory to be used. On the other hand the spin-orbit scattering, which is often rather weak, involves the derivative of the true potential which is much greater near the nucleus in comparison with the derivative of a pseudopotential. In addition the pseudo wave functions are really plane waves 
which are orthogonalized to the core states. For the moment we will treat $U_{0}(\boldsymbol{q})$ as a weak potential but in assuming the magnitude of spin-orbit interaction affects it in the final analysis, it is essential to bear in mind the true behaviour of the potential near the nucleus and the nature of the true wave functions. Equation (5) is equivalent to a set of four coupled equations for $\rho_{\alpha \alpha}(\boldsymbol{R}, \boldsymbol{K}), \rho_{\alpha \beta}(\boldsymbol{R}, \boldsymbol{K}), \rho_{\beta \alpha}(\boldsymbol{R}, \boldsymbol{K})$, and $\rho_{\beta \beta}(\boldsymbol{R}, \boldsymbol{K})$ so that the equations for the diagonal elements of the Wigner matrix are mutually coupled by the off-diagonal ones. Only the diagonal components of $\rho_{s^{\prime} s}(\boldsymbol{R}, \boldsymbol{K})$ will be required in our calculation of the current. The Eq. (5) can be simplified. The Hermitian property of the Wigner matrix operator enables the four equations to be reduced to two. Furthermore we can neglect the contribution coming from the term which includes the matrix element of the Pauli operator between identical spin states as this can be regarded as incorporated into the spin independent potential. Finally we will neglect the source term in the equations for the off-diagonal elements of the Wigner matrix and the spin contribution to the current operator, as we are primarily concerned with the dynamics of the electrons described by the lefthand side of the equations. The point is that spin-orbit effects are often weak except in cases like QI effects and indeed are not important in the source terms in the equations. The simplified equations are therefore

$$
\begin{aligned}
& {\left[\frac{\hbar \boldsymbol{K}}{m} \cdot \nabla+\varepsilon\right] \rho_{\alpha \alpha}(\boldsymbol{R}, \boldsymbol{K})+\frac{2}{\hbar} \sum_{q} \mathfrak{I}\left\{U_{0}(\boldsymbol{q}) \mathrm{e}^{i q \cdot \boldsymbol{R}}\right\} \rho_{\alpha \alpha}\left(\boldsymbol{R}, \boldsymbol{K}+\frac{1}{2} \boldsymbol{q}\right)} \\
& -\frac{2}{\hbar} \sum_{q} \frac{b_{\mathrm{SO}}}{2} \mathfrak{I}\left\{\hat{\Lambda}(\boldsymbol{q}, \boldsymbol{K}) \cdot \sigma_{\beta \alpha}\right\} \rho_{\alpha \beta}\left(\boldsymbol{R}, \boldsymbol{K}+\frac{1}{2} \boldsymbol{q}\right) \\
& =-e \boldsymbol{E}\left[\frac{\hbar \boldsymbol{K}}{m}+\boldsymbol{v}_{\alpha \alpha}^{S O}(\boldsymbol{K})\right] \phi(\boldsymbol{R}, \boldsymbol{K}),
\end{aligned}
$$

and

$$
\begin{aligned}
& {\left[\frac{\hbar \boldsymbol{K}}{m} \cdot \nabla+\varepsilon\right] \rho_{\alpha \beta}(\boldsymbol{R}, \boldsymbol{K})+\frac{2}{\hbar} \sum_{q} \mathfrak{I}\left\{U_{0}(\boldsymbol{q}) \mathrm{e}^{i q \cdot R}\right\} \rho_{\alpha \beta}\left(\boldsymbol{R}, \boldsymbol{K}+\frac{1}{2} \boldsymbol{q}\right)} \\
& -\frac{2}{\hbar} \sum_{\boldsymbol{q}} \frac{b_{\mathrm{SO}}}{2} \mathfrak{I}\left\{\hat{\Lambda}(\boldsymbol{q}, \boldsymbol{K}) \cdot \sigma_{\alpha \beta}\right\} \rho_{\alpha \alpha}\left(\boldsymbol{R}, \boldsymbol{K}+\frac{1}{2} \boldsymbol{q}\right)=0,
\end{aligned}
$$

which still constitute a formidable task to solve in a reasonable fashion.

One of the possible ways to proceed further is by using a projection operator method like that of Zwanzig [44] and Mori [45]. We divide each function of variables $(\boldsymbol{R}, \boldsymbol{K})$ into two parts as follows

$$
f_{s^{\prime} s}(\boldsymbol{R}, \boldsymbol{K})=f_{s^{\prime} s}^{A}(\boldsymbol{k})+f_{s^{\prime} s}^{F}(\boldsymbol{R}, \boldsymbol{K}),
$$

where $f_{s^{\prime} s}^{A}(\boldsymbol{K})$ is the average part defined by $\hat{A}\left\{f_{s^{\prime} s}(\boldsymbol{R}, \boldsymbol{K})\right\}=\Omega^{-1} \int d^{3} R f_{s^{\prime} s}(\boldsymbol{R}, \boldsymbol{K})$ and $f_{s^{\prime} s}^{F}(\boldsymbol{R}, \boldsymbol{K})$ is the fluctuating part defined by $\hat{F}\left\{f_{s^{\prime} s}(\boldsymbol{R}, \boldsymbol{K})\right\}=[1-\hat{A}]\left\{f_{s^{\prime} s}(\boldsymbol{R}, \boldsymbol{K})\right\} . \hat{A}$ denotes the averaging operator, $\Omega$ is the volume of the system, and $\hat{F}$ is the fluctuating operator, which describes the deviation from the average value and therefore is space dependent. We now separate the elements of the Wigner matrix and the source function in Eqs. (7) and (8) into the average and fluctuating part according to the formula (9). If we act with the operator $\hat{A}$ on the Eq. (7), and then with the operator $\hat{F}$ we obtain a coupled pair of equations. This is the approach used by Morgan et al. [26, 46] but further complicated by the spin indices. The result is

$$
\begin{aligned}
\varepsilon \rho_{\alpha \alpha}^{A}(\boldsymbol{K}) & +\hat{A}\left\{\frac{2}{\hbar} \sum_{q}\left[\mathfrak{I}\left\{U_{0}(\boldsymbol{q}) \mathrm{e}^{i q \cdot \boldsymbol{R}}\right\} \rho_{\alpha \alpha}^{F}\left(\boldsymbol{R}, \boldsymbol{K}+\frac{1}{2} \boldsymbol{q}\right)-\frac{b_{\mathrm{SO}}}{2} \mathfrak{I}\left\{\hat{\Lambda}(\boldsymbol{q}, \boldsymbol{K}) \cdot \sigma_{\beta \alpha}\right\} \rho_{\alpha \beta}^{F}\left(\boldsymbol{R}, \boldsymbol{K}+\frac{1}{2} \boldsymbol{q}\right)\right]\right\} \\
& =-e \boldsymbol{E} \cdot \boldsymbol{v}_{\alpha \alpha}(\boldsymbol{K}) \phi^{A}(\boldsymbol{K})
\end{aligned}
$$


and

$$
\begin{aligned}
& \hat{F}\left\{\left[\frac{\hbar \boldsymbol{K}}{m} \cdot \nabla+\varepsilon\right] \rho_{\alpha \alpha}^{F}(\boldsymbol{R}, \boldsymbol{K})\right\}+\hat{F}\left\{\frac { 2 } { \hbar } \sum _ { q } \left[\mathfrak{I}\left\{U_{0}(\boldsymbol{q}) \mathrm{e}^{i \boldsymbol{q} \cdot \boldsymbol{R}}\right\} \rho_{\alpha \alpha}^{F}\left(\boldsymbol{R}, \boldsymbol{K}+\frac{1}{2} \boldsymbol{q}\right)\right.\right. \\
& \left.\left.-\frac{b_{\mathrm{SO}}}{2} \mathfrak{I}\left\{\hat{\Lambda}(\boldsymbol{q}, \boldsymbol{K}) \cdot \sigma_{\beta \alpha}\right\} \rho_{\alpha \beta}^{F}\left(\boldsymbol{R}, \boldsymbol{K}+\frac{1}{2} \boldsymbol{q}\right)\right]\right\} \\
& =-e \boldsymbol{E} \cdot \boldsymbol{v}_{\alpha \alpha}(\boldsymbol{K}) \phi^{F}(\boldsymbol{R}, \boldsymbol{K})-\frac{2}{\hbar} \sum_{q}\left[\mathfrak{I}\left\{U_{0}(\boldsymbol{q}) \mathrm{e}^{i \boldsymbol{q} \cdot \boldsymbol{R}}\right\} \rho_{\alpha \alpha}^{A}\left(\boldsymbol{K}+\frac{1}{2} \boldsymbol{q}\right)+\frac{b_{\mathrm{SO}}}{2} \mathfrak{I}\left\{\hat{\Lambda}(\boldsymbol{q}, \boldsymbol{K}) \cdot \sigma_{\beta \alpha}\right\} \rho_{\alpha \beta}^{A}\left(\boldsymbol{K}+\frac{1}{2} \boldsymbol{q}\right)\right] \text {, }
\end{aligned}
$$

where $\boldsymbol{v}_{\alpha \alpha}(\boldsymbol{K})$ is the usual velocity, equal to $\hbar \boldsymbol{K} / m$, while the spin-orbit part of the velocity is neglected as this is not important in the present context. This non-classical contribution stems from the commutator of the position operator with the spin-orbit Hamiltonian.

In the same way as for Eq. (8) we obtain

$$
\begin{aligned}
\varepsilon \rho_{\alpha \beta}^{A}(\boldsymbol{K})+\hat{A}\{ & \frac{2}{\hbar} \sum_{q}\left[\mathfrak{I}\left\{U_{0}(\boldsymbol{q}) \mathrm{e}^{i \boldsymbol{q} \cdot \boldsymbol{R}}\right\} \rho_{\alpha \alpha}^{F}\left(\boldsymbol{R}, \boldsymbol{K}+\frac{1}{2} \boldsymbol{q}\right)-\frac{b_{\mathrm{SO}}}{2} \mathfrak{I}\left\{\hat{\Lambda}(\boldsymbol{q}, \boldsymbol{K}) \cdot \sigma_{\alpha \beta}\right\}\right. \\
& \left.\left.\times \rho_{\alpha \alpha}^{F}\left(\boldsymbol{R}, \boldsymbol{K}+\frac{1}{2} \boldsymbol{q}\right)\right]\right\}=0,
\end{aligned}
$$

and

$$
\begin{aligned}
\hat{F}\left\{\left[\frac{\hbar \boldsymbol{K}}{m} \cdot \nabla+\varepsilon\right] \rho_{\alpha \beta}^{F}(\boldsymbol{R}, \boldsymbol{K})\right\}+\hat{F}\{ & \frac{2}{\hbar} \sum_{\boldsymbol{q}}\left[\mathfrak{I}\left\{U_{0}(\boldsymbol{q}) \mathrm{e}^{i \boldsymbol{q} \cdot \boldsymbol{R}}\right\} \rho_{\alpha \beta}^{F}\left(\boldsymbol{R}, \boldsymbol{K}+\frac{1}{2} \boldsymbol{q}\right)\right. \\
& \left.\left.\quad-\frac{b_{\mathrm{SO}}}{2} \mathfrak{I}\left\{\hat{\Lambda}(\boldsymbol{q}, \boldsymbol{K}) \cdot \sigma_{\alpha \beta}\right\} \rho_{\alpha \alpha}^{F}\left(\boldsymbol{R}, \boldsymbol{K}+\frac{1}{2} \boldsymbol{q}\right)\right]\right\} \\
=-\frac{2}{\hbar} \sum_{\boldsymbol{q}}\left[\mathfrak{I}\left\{U_{0}(\boldsymbol{q}) \mathrm{e}^{i \boldsymbol{q} \cdot \boldsymbol{R}}\right\} \rho_{\alpha \beta}^{A}(\boldsymbol{K}\right. & \left.\left.+\frac{1}{2} \boldsymbol{q}\right)-\frac{b_{S O}}{2} \mathfrak{I}\left\{\hat{\Lambda}(\boldsymbol{q}, \boldsymbol{K}) \cdot \sigma_{\alpha \beta}\right\} \rho_{\alpha \alpha}^{A}\left(\boldsymbol{K}+\frac{1}{2} \boldsymbol{q}\right)\right] .
\end{aligned}
$$

The Eqs. (11) and (13) can be solved formally by finding the inverse operator to obtain the fluctuating parts $\rho_{\alpha \alpha}^{F}(\boldsymbol{R}, \boldsymbol{K})$ and $\rho_{\alpha \beta}^{F}(\boldsymbol{R}, \boldsymbol{K})$ in terms of the average parts $\rho_{\alpha \alpha}^{A}(\boldsymbol{K})$ and $\rho_{\alpha \beta}^{A}(\boldsymbol{K})$ but of course we must make simplifying approximations to make progress. We further neglect the spin-orbit interaction terms in the fluctuating part on the left-hand side of Eqs. (11) and (13), but we retain them in the average part of the right-hand side of these equations. This is because these terms are off-diagonal in both spin space and $\boldsymbol{K}$-space and we are really developing a type of self-consistent perturbation theory for the offdiagonal parts in terms of diagonal parts. We then obtain using the methods described in [26, 46]

$$
\begin{aligned}
\rho_{\alpha \alpha}^{F}(\boldsymbol{R}, \boldsymbol{K})=\sum_{\boldsymbol{K}^{\prime}} \int d^{3} R^{\prime} G^{F}\left(\boldsymbol{R}, \boldsymbol{R}^{\prime}, \boldsymbol{K}, \boldsymbol{K}^{\prime}\right)[ & \left.-e \boldsymbol{E} \cdot \boldsymbol{v}_{\alpha \alpha}\left(\boldsymbol{K}^{\prime}\right) \phi^{F}\left(\boldsymbol{R}^{\prime}, \boldsymbol{K}^{\prime}\right)\right] \\
+ & \sum_{\boldsymbol{K}^{\prime}} \int d^{3} R^{\prime} G^{F}\left(\boldsymbol{R}, \boldsymbol{R}^{\prime}, \boldsymbol{K}, \boldsymbol{K}^{\prime}\right)\{ \\
- & -\frac{2}{\hbar} \sum_{\boldsymbol{q}}\left[\mathfrak{I}\left\{U_{0}(\boldsymbol{q}) \mathrm{e}^{i \boldsymbol{q} \cdot \boldsymbol{R}^{\prime}}\right\} \rho_{\alpha \alpha}^{A}\left(\boldsymbol{K}^{\prime}+\frac{1}{2} \boldsymbol{q}\right)\right. \\
& \left.\left.+\frac{b_{\mathrm{SO}}}{2} \mathfrak{I}\left\{\hat{\Lambda}\left(\boldsymbol{q}, \boldsymbol{K}^{\prime}\right) \cdot \sigma_{\beta \alpha}\right\} \rho_{\alpha \beta}^{A}\left(\boldsymbol{K}^{\prime}+\frac{1}{2} \boldsymbol{q}\right)\right]\right\}, \\
\rho_{\alpha \beta}^{F}(\boldsymbol{R}, \boldsymbol{K})=\sum_{\boldsymbol{K}^{\prime}} \int d^{3} R^{\prime} G^{F}\left(\boldsymbol{R}, \boldsymbol{R}^{\prime}, \boldsymbol{K}, \boldsymbol{K}^{\prime}\right)\{ & -\frac{2}{\hbar} \sum_{q}\left[\mathfrak{I}\left\{U_{0}(\boldsymbol{q}) \mathrm{e}^{i \boldsymbol{q} \cdot \boldsymbol{R}^{\prime}}\right\} \rho_{\alpha \beta}^{A}\left(\boldsymbol{K}^{\prime}+\frac{1}{2} \boldsymbol{q}\right)\right. \\
& \left.\left.+\frac{b_{\mathrm{SO}}}{2} \mathfrak{I}\left\{\hat{\Lambda}\left(\boldsymbol{q}, \boldsymbol{K}^{\prime}\right) \cdot \sigma_{\alpha \beta}\right\} \rho_{\alpha \alpha}^{A}\left(\boldsymbol{K}^{\prime}+\frac{1}{2} \boldsymbol{q}\right)\right]\right\},
\end{aligned}
$$


where $G^{F}$ is a Green function which is an inverse of the operator acting on the left-hand side of the Eqs. (11) and (13) when the fluctuating part of the spin-orbit term is neglected. At a later stage this Green function will be obtained with an effective medium approximation as described in [28] and renders the equations in a form which can be further simplified. It should be emphasized that it is not a oneparticle coherent potential type of approximation as it relates to the density matrix, and not a one-particle Green function of the Schrödinger equation [47] which is the reason why weak and strong localization can be considered within this framework. These solutions can be substituted into (10) and (12), and after some rearrangement we obtain the final form for the transport equations

$\varepsilon \rho_{\alpha \alpha}^{A}(\boldsymbol{K})+\sum_{\boldsymbol{K}^{\prime}}\left[T_{0}\left(\boldsymbol{K}, \boldsymbol{K}^{\prime}\right)+T_{\alpha \beta, \beta \alpha}\left(\boldsymbol{K}, \boldsymbol{K}^{\prime \prime}\right)\right]\left[\rho_{\alpha \alpha}^{A}(\boldsymbol{K})-\rho_{\alpha \alpha}^{A}\left(\boldsymbol{K}^{\prime}\right)\right]=-\frac{1}{3} e \boldsymbol{E} \cdot \boldsymbol{v}_{\alpha \alpha}(\boldsymbol{K})\left[1+\gamma_{0}(\boldsymbol{K})\right] \phi^{A}(\boldsymbol{K})$,

and

$$
\begin{aligned}
& \varepsilon \rho_{\alpha \beta}^{A}(\boldsymbol{K})+\sum_{\boldsymbol{K}^{\prime}}\left\{\left[T_{0}\left(\boldsymbol{K}, \boldsymbol{K}^{\prime}\right)+T_{\alpha \beta, \beta \alpha}\left(\boldsymbol{K}, \boldsymbol{K}^{\prime}\right)\right]\left[\rho_{\alpha \beta}^{A}(\boldsymbol{K})-\rho_{\alpha \beta}^{A}\left(\boldsymbol{K}^{\prime}\right)\right]-2 T_{0, \alpha \beta}\left(\boldsymbol{K}, \boldsymbol{K}^{\prime}\right)\left[\rho_{\alpha \alpha}^{A}(\boldsymbol{K})-\rho_{\alpha \alpha}^{A}\left(\boldsymbol{K}^{\prime}\right)\right]\right\} \\
& =-e \boldsymbol{E} \cdot \boldsymbol{v}_{\alpha \alpha}(\boldsymbol{K})\left[1+\gamma_{\alpha \beta}(\boldsymbol{k})\right] \phi^{A}(\boldsymbol{K})
\end{aligned}
$$

where $T\left(\boldsymbol{K}, \boldsymbol{K}^{\prime}\right)$ is a generalized scattering kernel which describes the scattering of the electrons and replaces the usual scattering probability in the normal Boltzmann equation. The function $\gamma(\boldsymbol{K})$ modifies the injection of electrons into the system by the electric field taking into account the fluctuations in the Wigner representation of the Fermi-Dirac distribution function [26]. Below we now present the definitions of all the $T$-terms and $\gamma$-terms which exist in the transport equations. It should be noted that $T$ does $n o t$ denote the $t$-matrix of the system. It describes multiple scattering within the system as opposed to the scattering of an incident $\boldsymbol{K}$-state on a finite system. The terms in (16) and (17) are defined by

$$
\begin{aligned}
& T_{0}\left(\boldsymbol{K}, \boldsymbol{K}^{\prime}\right)=\frac{1}{\Omega}\left(\frac{2}{\hbar}\right)^{2} \sum_{q} \sum_{q^{\prime}} \int d^{3} R d^{3} R^{\prime} \hat{P}(\boldsymbol{q}) G^{F}\left(\boldsymbol{R}, \boldsymbol{R}^{\prime}, \boldsymbol{K}+\frac{1}{2} \boldsymbol{q}, \boldsymbol{K}^{\prime}-\frac{1}{2} \boldsymbol{q}^{\prime}\right) \hat{P}\left(\boldsymbol{q}^{\prime}\right), \\
& T_{\alpha \beta, \beta \alpha}\left(\boldsymbol{K}, \boldsymbol{K}^{\prime}\right)=\frac{1}{\Omega}\left(\frac{2}{\hbar}\right)^{2} \sum_{q} \sum_{q^{\prime}} \int d^{3} R d^{3} R^{\prime} \hat{O}_{\alpha \beta}(\boldsymbol{q}) G^{F}\left(\boldsymbol{R}, \boldsymbol{R}^{\prime}, \boldsymbol{K}+\frac{1}{2} \boldsymbol{q}, \boldsymbol{K}^{\prime}-\frac{1}{2} \boldsymbol{q}^{\prime}\right) \hat{O}_{\beta \alpha}\left(\boldsymbol{q}^{\prime}\right), \\
& T_{0, \beta \alpha}\left(\boldsymbol{K}, \boldsymbol{K}^{\prime}\right)=\frac{1}{\Omega}\left(\frac{2}{\hbar}\right)^{2} \sum_{q} \sum_{q^{\prime}} \int d^{3} R d^{3} R^{\prime} \hat{P}(\boldsymbol{q}) G^{F}\left(\boldsymbol{R}, \boldsymbol{R}^{\prime}, \boldsymbol{K}+\frac{1}{2} \boldsymbol{q}, \boldsymbol{K}^{\prime}-\frac{1}{2} \boldsymbol{q}^{\prime}\right) \hat{O}_{\beta \alpha}\left(\boldsymbol{q}^{\prime}\right) \\
& \gamma_{0}(\boldsymbol{K})=-\frac{1}{\Omega} \frac{2}{\hbar} \sum_{\boldsymbol{K}^{\prime}} \sum_{q} \int d^{3} R d^{3} R^{\prime} \hat{P}(\boldsymbol{q}) G^{F}\left(\boldsymbol{R}, \boldsymbol{R}^{\prime}, \boldsymbol{K}, \boldsymbol{K}^{\prime}\right) \frac{v_{\alpha \alpha}\left(K_{x}^{\prime}\right)}{v_{\alpha \alpha}\left(K_{x}\right)} \frac{\phi\left(\boldsymbol{R}^{\prime}, \boldsymbol{K}^{\prime}\right)-\phi^{A}\left(\boldsymbol{K}^{\prime}\right)}{\phi^{A}(\boldsymbol{K})} \\
& \gamma_{\alpha \beta}(\boldsymbol{K})=-\frac{1}{\Omega} \frac{2}{\hbar} \sum_{K^{\prime}} \sum_{q} \int d^{3} R d^{3} R^{\prime} \hat{O}_{\alpha \beta}(\boldsymbol{q}) G^{F}\left(\boldsymbol{R}, \boldsymbol{R}^{\prime}, \boldsymbol{K}, \boldsymbol{K}^{\prime}\right) \frac{v_{\alpha \alpha}\left(K_{x}^{\prime}\right)}{v_{\alpha \alpha}\left(K_{x}\right)} \frac{\phi\left(\boldsymbol{R}^{\prime}, \boldsymbol{K}^{\prime}\right)-\phi^{A}\left(\boldsymbol{K}^{\prime}\right)}{\phi^{A}(\boldsymbol{K})}
\end{aligned}
$$

and the operators $\hat{P}(\boldsymbol{q}), \hat{O}_{\alpha \beta}(\boldsymbol{q}), \hat{O}_{\beta \alpha}(\boldsymbol{q})$ are defined as follows

$$
\begin{aligned}
& \hat{P}(\boldsymbol{q})=\mathfrak{I}\left\{U_{0}(\boldsymbol{q}) \mathrm{e}^{\mathrm{i} \boldsymbol{q} \cdot \boldsymbol{R}}\right\}, \\
& \hat{O}_{\alpha \beta}(\boldsymbol{q})=\frac{b_{\mathrm{SO}}}{2} \mathfrak{I}\left\{\hat{\Lambda}(\boldsymbol{q}, \boldsymbol{K}) \cdot \sigma_{\alpha \beta}\right\}, \\
& \hat{O}_{\beta \alpha}(\boldsymbol{q})=\frac{b_{\mathrm{SO}}}{2} \mathfrak{I}\left\{\hat{\Lambda}(\boldsymbol{q}, \boldsymbol{K}) \cdot \sigma_{\beta \alpha}\right\} .
\end{aligned}
$$

The operator $T_{0}\left(\boldsymbol{K}, \boldsymbol{K}^{\prime}\right)$ given by the Eq. (18) is responsible for the spin-independent scattering which at the lowest level of approximation simply corresponds to the Born approximation. The operator defined as $T_{\alpha \beta, \beta \alpha}\left(\boldsymbol{K}, \boldsymbol{K}^{\prime}\right)$ is the spin-flip scattering part, and $T_{0, \beta \alpha}\left(\boldsymbol{K}, \boldsymbol{K}^{\prime}\right)$ comes from the cross-product of the spin-orbit potential and ordinary potential which in simpler situations is termed the skew scattering [48]. 
Perhaps this term could be important in general and it would be simple to incorporate in future work but here it plays no significant role because it vanishes at the level of approximation used here. We can ignore Eq. (17) for the off-diagonal elements of the Wigner matrix because the current involving the usual velocity does not depend on the off-diagonal elements. We will now focus on the problem of constructing an approximation for the generalized scattering operator $T\left(\boldsymbol{K}, \boldsymbol{K}^{\prime}\right)$ because this is the most important quantity in the context of quantum interference.

\section{Generalized scattering kernel}

We will now use an effective medium approximation for the density matrix as in the original paper [26, $46]$ to determine the analytical form of the generalized scattering kernel. The main idea of this approximation is based on the following picture. The disordered system is replaced by a medium, the properties of which are determined by two quantities: $T\left(\boldsymbol{K}, \boldsymbol{K}^{\prime}\right)$ and $\gamma(\boldsymbol{K})$. A scatterer is embedded in this medium and $T\left(\boldsymbol{K}, \boldsymbol{K}^{\prime}\right)$ and $\gamma(\boldsymbol{K})$ must be determined self-consistently. Again it must be emphasized that this much more complex task than a coherent potential approximation [49] for a one-particle Green function of the Schrödinger equation. Perhaps it is appropriate to note that in this theory we need in general the two quantities $T\left(\boldsymbol{K}, \boldsymbol{K}^{\prime}\right)$ and $\gamma(\boldsymbol{K})$, whereas the use of the Kubo formula requires two-particle Green function for the particle-particle and particle-hole channel $[10,25]$ so that both approaches have their respective merits. The inclusion of $\gamma$ in general is important to ensure the convergence of integral as is the inclusion of both types of two particle Green functions [50].

In (15) the problem of determining $G^{F}$ is solved by truncating an infinite hierarchy of equations formed by taking configurational averages of the Wigner equation leading to closed equations for $T\left(\boldsymbol{K}, \boldsymbol{K}^{\prime}\right)$ and $\gamma(\boldsymbol{K})$. The configurational average of (7) and (8) gives

$$
\begin{aligned}
& {\left[\frac{\hbar \boldsymbol{K}}{m} \cdot \nabla+\varepsilon\right] \bar{\rho}_{\alpha \alpha}(\boldsymbol{R}, \boldsymbol{K})+\frac{2}{\hbar} \sum_{q}\left[\frac{N}{\Omega} \mathfrak{I}\left\{\int d^{3} R^{\prime} d^{3} r_{1} p\left(\boldsymbol{r}_{1}\right) u_{a}\left(\boldsymbol{R}^{\prime}-\boldsymbol{r}_{1}\right) \mathrm{e}^{i \boldsymbol{q} \cdot\left(\boldsymbol{R}-\boldsymbol{R}^{\prime}\right)}\right\} \bar{\rho}_{\alpha \alpha}\left(\boldsymbol{R}, \boldsymbol{K}+\frac{1}{2} \boldsymbol{q} \mid \boldsymbol{r}_{1}\right)\right.} \\
& \left.-\frac{b_{\mathrm{SO}}}{2} \frac{N}{\Omega} \mathfrak{I}\left\{\int d^{3} R^{\prime} d^{3} r_{1} p\left(\boldsymbol{r}_{1}\right) u_{a}\left(\boldsymbol{R}^{\prime}-\boldsymbol{r}_{1}\right) \mathrm{e}^{i \boldsymbol{q} \cdot\left(\boldsymbol{R}-\boldsymbol{R}^{\prime}\right)} \boldsymbol{\kappa} \cdot \sigma_{\beta \alpha}\right\} \bar{\rho}_{\alpha \beta}\left(\boldsymbol{R}, \boldsymbol{K}+\frac{1}{2} \boldsymbol{q} \mid \boldsymbol{r}_{1}\right)\right]=-e \boldsymbol{E} \cdot \boldsymbol{v}_{\alpha \alpha}(\boldsymbol{K}) \bar{\phi}(\boldsymbol{R}, \boldsymbol{K})
\end{aligned}
$$

and

$$
\begin{aligned}
& {\left[\frac{\hbar \boldsymbol{K}}{m} \cdot \nabla+\varepsilon\right] \bar{\rho}_{\alpha \beta}(\boldsymbol{R}, \boldsymbol{K})+\frac{2}{\hbar} \sum_{q}\left[\frac{N}{\Omega} \mathfrak{I}\left\{\int d^{3} R^{\prime} d^{3} r_{1} p\left(\boldsymbol{r}_{1}\right) u_{a}\left(\boldsymbol{R}^{\prime}-\boldsymbol{r}_{1}\right) \mathrm{e}^{i \boldsymbol{q} \cdot\left(\boldsymbol{R}-\boldsymbol{R}^{\prime}\right)}\right\} \bar{\rho}_{\alpha \beta}\left(\boldsymbol{R}, \boldsymbol{K}+\frac{1}{2} \boldsymbol{q} \mid \boldsymbol{r}_{1}\right)\right.} \\
& \left.-\frac{b_{\mathrm{SO}}}{2} \frac{N}{\Omega} \mathfrak{I}\left\{\int d^{3} R^{\prime} d^{3} r_{1} p\left(\boldsymbol{r}_{1}\right) u_{a}\left(\boldsymbol{R}^{\prime}-\boldsymbol{r}_{1}\right) \mathrm{e}^{i \boldsymbol{q} \cdot\left(\boldsymbol{R}-\boldsymbol{R}^{\prime}\right)} \boldsymbol{\kappa} \cdot \sigma_{\beta \alpha}\right\} \bar{\rho}_{\alpha \alpha}\left(\boldsymbol{R}, \boldsymbol{K}+\frac{1}{2} \boldsymbol{q} \mid \boldsymbol{r}_{1}\right)\right]=0,
\end{aligned}
$$

respectively, where $\boldsymbol{\kappa}=\boldsymbol{q} \times(\nabla-2 i \boldsymbol{K}), p\left(\boldsymbol{r}_{1}\right)$ is the probability of finding an ion at $\boldsymbol{r}_{1}$ and $\bar{\rho}_{s^{\prime} s}\left(\boldsymbol{R}, \boldsymbol{K} \mid \boldsymbol{r}_{1}\right)$ is the distribution function when a scatterer is fixed at $\boldsymbol{r}_{1}$. If we compare these equations with Eqs. (10) and (12), we see that the fluctuating part of the Wigner matrix $\rho_{s^{\prime} s}^{F}(\boldsymbol{R}, \boldsymbol{K})$ is replaced by $\bar{\rho}_{s^{\prime} s}\left(\boldsymbol{R}, \boldsymbol{K} \mid \boldsymbol{r}_{1}\right)$.

The next equations in the hierarchy are

$$
\begin{aligned}
& {\left[\frac{\hbar \boldsymbol{K}}{m} \cdot \nabla+\varepsilon\right] \bar{\rho}_{\alpha \alpha}\left(\boldsymbol{R}, \boldsymbol{K} \mid \boldsymbol{r}_{1}\right)+\frac{2}{\hbar} \sum_{q}\left[\mathfrak{I}\left\{\int d^{3} R^{\prime} u_{a}\left(\boldsymbol{R}^{\prime}-\boldsymbol{r}_{1}\right) \mathrm{e}^{i \boldsymbol{q} \cdot\left(\boldsymbol{R}-\boldsymbol{R}^{\prime}\right)}\right\} \bar{\rho}_{\alpha \alpha}\left(\boldsymbol{R}, \boldsymbol{K}+\frac{1}{2} \boldsymbol{q} \mid \boldsymbol{r}_{1}\right)\right.} \\
& \left.-\frac{b_{\mathrm{SO}}}{2} \mathfrak{I}\left\{\int d^{3} R^{\prime} u_{a}\left(\boldsymbol{R}^{\prime}-\boldsymbol{r}_{1}\right) \mathrm{e}^{i \boldsymbol{q} \cdot\left(\boldsymbol{R}-\boldsymbol{R}^{\prime}\right)} \kappa \cdot \sigma_{\beta \alpha}\right\} \bar{\rho}_{\alpha \beta}\left(\boldsymbol{R}, \boldsymbol{K}+\frac{1}{2} \boldsymbol{q} \mid \boldsymbol{r}_{1}\right)\right] \\
& +\frac{2}{\hbar} \sum_{q}\left[\frac{N-1}{\Omega} \mathfrak{I}\left\{\int d^{3} R^{\prime} d^{3} r_{2} p\left(\boldsymbol{r}_{2} \mid \boldsymbol{r}_{1}\right) u_{a}\left(\boldsymbol{R}^{\prime}-\boldsymbol{r}_{2}\right) \mathrm{e}^{i \boldsymbol{q} \cdot\left(\boldsymbol{R}-\boldsymbol{R}^{\prime}\right)}\right\} \bar{\rho}_{\alpha \alpha}\left(\boldsymbol{R}, \boldsymbol{K}+\frac{1}{2} \boldsymbol{q} \mid \boldsymbol{r}_{1} \boldsymbol{r}_{2}\right)\right. \\
& \left.-\frac{b_{\mathrm{SO}}}{2} \frac{N-1}{\Omega} \mathfrak{I}\left\{\int d^{3} R^{\prime} d^{3} r_{2} p\left(\boldsymbol{r}_{2} \mid \boldsymbol{r}_{1}\right) u_{a}\left(\boldsymbol{R}^{\prime}-\boldsymbol{r}_{2}\right) \mathrm{e}^{i \boldsymbol{q} \cdot\left(\boldsymbol{R}-\boldsymbol{R}^{\prime}\right)} \kappa \cdot \sigma_{\beta \alpha}\right\} \bar{\rho}_{\alpha \beta}\left(\boldsymbol{R}, \boldsymbol{K}+\frac{1}{2} \boldsymbol{q} \mid \boldsymbol{r}_{1} \boldsymbol{r}_{2}\right)\right] \\
& =-e \boldsymbol{E} \cdot \boldsymbol{v}_{\alpha \alpha}(\boldsymbol{K}) \bar{\phi}\left(\boldsymbol{R}, \boldsymbol{K} \mid \boldsymbol{r}_{1}\right)
\end{aligned}
$$


and

$$
\begin{aligned}
& {\left[\frac{\hbar \boldsymbol{K}}{m} \cdot \nabla+\varepsilon\right] \bar{\rho}_{\alpha \beta}\left(\boldsymbol{R}, \boldsymbol{K} \mid \boldsymbol{r}_{1}\right)+\frac{2}{\hbar} \sum_{\boldsymbol{q}}\left[\mathfrak{I}\left\{\int d^{3} R^{\prime} u_{a}\left(\boldsymbol{R}^{\prime}-\boldsymbol{r}_{1}\right) \mathrm{e}^{i \boldsymbol{q} \cdot\left(\boldsymbol{R}-\boldsymbol{R}^{\prime}\right)}\right\} \bar{\rho}_{\alpha \beta}\left(\boldsymbol{R}, \boldsymbol{K}+\frac{1}{2} \boldsymbol{q} \mid \boldsymbol{r}_{1}\right)\right.} \\
& \left.-\frac{b_{\mathrm{SO}}}{2} \frac{N}{\Omega} \mathfrak{I}\left\{\int d^{3} R^{\prime} u_{a}\left(\boldsymbol{R}^{\prime}-\boldsymbol{r}_{1}\right) \mathrm{e}^{i \boldsymbol{q} \cdot\left(\boldsymbol{R}-\boldsymbol{R}^{\prime}\right)} \boldsymbol{\kappa} \cdot \sigma_{\alpha \beta}\right\} \bar{\rho}_{\alpha \alpha}\left(\boldsymbol{R}, \boldsymbol{K}+\frac{1}{2} \boldsymbol{q} \mid \boldsymbol{r}_{1}\right)\right] \\
& +\frac{2}{\hbar} \sum_{q}\left[\frac{N-1}{\Omega} \mathfrak{I}\left\{\int d^{3} R^{\prime} d^{3} r_{2} p\left(\boldsymbol{r}_{2} \mid \boldsymbol{r}_{1}\right) u_{a}\left(\boldsymbol{R}^{\prime}-\boldsymbol{r}_{2}\right) \mathrm{e}^{i \boldsymbol{q} \cdot\left(\boldsymbol{R}-\boldsymbol{R}^{\prime}\right)}\right\} \bar{\rho}_{\alpha \beta}\left(\boldsymbol{R}, \boldsymbol{K}+\frac{1}{2} \boldsymbol{q} \mid \boldsymbol{r}_{1} \boldsymbol{r}_{2}\right)\right. \\
& \left.-\frac{b_{\mathrm{SO}}}{2} \frac{N-1}{\Omega} \mathfrak{I}\left\{\int d^{3} R^{\prime} d^{3} r_{2} p\left(\boldsymbol{r}_{2} \mid \boldsymbol{r}_{1}\right) u_{a}\left(\boldsymbol{R}^{\prime}-\boldsymbol{r}_{2}\right) \mathrm{e}^{i \boldsymbol{q} \cdot\left(\boldsymbol{R}-\boldsymbol{R}^{\prime}\right)} \kappa \cdot \sigma_{\alpha \beta}\right\} \bar{\rho}_{\alpha \alpha}\left(\boldsymbol{R}, \boldsymbol{K}+\frac{1}{2} \boldsymbol{q} \mid \boldsymbol{r}_{1} \boldsymbol{r}_{2}\right)\right]=0,
\end{aligned}
$$

where $\bar{\rho}_{s^{\prime} s}\left(\boldsymbol{R}, \boldsymbol{K} \mid \boldsymbol{r}_{1} \boldsymbol{r}_{2}\right)$ corresponds to two scatterers fixed at $\boldsymbol{r}_{1}$ and $\boldsymbol{r}_{2}$, and $p\left(\boldsymbol{r}_{2} \mid \boldsymbol{r}_{1}\right)$ is the pair distribution function. Writing $\bar{\rho}_{s^{\prime} s}\left(\boldsymbol{R}, \boldsymbol{K} \mid \boldsymbol{r}_{1} \boldsymbol{r}_{2}\right)=\bar{\rho}_{s^{\prime} s}\left(\boldsymbol{R}, \boldsymbol{K} \mid \boldsymbol{r}_{1}\right)+\Delta \rho_{s^{\prime} s}\left(\boldsymbol{R}, \boldsymbol{K} \mid \boldsymbol{r}_{1} \boldsymbol{r}_{2}\right)$ in (28) and (29) we truncate the hierarchy of equations by replacing (28) and (29) by

$$
\begin{aligned}
& {\left[\frac{\hbar \boldsymbol{K}}{m} \cdot \nabla+\varepsilon\right] \bar{\rho}_{\alpha \alpha}\left(\boldsymbol{R}, \boldsymbol{K} \mid \boldsymbol{r}_{1}\right)+\frac{2}{\hbar} \sum_{q}\left[\mathfrak{I}\left\{\int d^{3} R^{\prime} \tilde{u}_{a}\left(\boldsymbol{R}^{\prime}-\boldsymbol{r}_{1}\right) \mathrm{e}^{i \boldsymbol{q} \cdot\left(\boldsymbol{R}-\boldsymbol{R}^{\prime}\right)}\right\} \bar{\rho}_{\alpha \alpha}\left(\boldsymbol{R}, \boldsymbol{K}+\frac{1}{2} \boldsymbol{q} \mid \boldsymbol{r}_{1}\right)\right.} \\
& \left.-\frac{b_{\mathrm{SO}}}{2} \mathfrak{I}\left\{\int d^{3} R^{\prime} \tilde{u}_{a}\left(\boldsymbol{R}^{\prime}-\boldsymbol{r}_{1}\right) \mathrm{e}^{i \boldsymbol{q} \cdot\left(\boldsymbol{R}-\boldsymbol{R}^{\prime}\right)} \kappa \cdot \sigma_{\beta \alpha}\right\} \bar{\rho}_{\alpha \beta}\left(\boldsymbol{R}, \boldsymbol{K}+\frac{1}{2} \boldsymbol{q} \mid \boldsymbol{r}_{1}\right)\right] \\
& +\sum_{\boldsymbol{K}^{\prime}}\left\{T_{0}\left(\boldsymbol{K}, \boldsymbol{K}^{\prime}\right)\left[\bar{\rho}_{\alpha \alpha}\left(\boldsymbol{R}, \boldsymbol{K} \mid \boldsymbol{r}_{1}\right)-\bar{\rho}_{\alpha \alpha}\left(\boldsymbol{R}, \boldsymbol{K}^{\prime} \mid \boldsymbol{r}_{1}\right)\right]\right. \\
& \left.-T_{\mathrm{SO}}\left(\boldsymbol{K}, \boldsymbol{K}^{\prime}\right)\left[\bar{\rho}_{\alpha \beta}\left(\boldsymbol{R}, \boldsymbol{K} \mid \boldsymbol{r}_{1}\right)-\bar{\rho}_{\alpha \beta}\left(\boldsymbol{R}, \boldsymbol{K}^{\prime} \mid \boldsymbol{r}_{1}\right)\right]\right\}=-e \boldsymbol{E} \cdot \boldsymbol{v}_{\alpha \alpha}(\boldsymbol{K}) \bar{\phi}\left(\boldsymbol{R}, \boldsymbol{K} \mid \boldsymbol{r}_{1}\right)
\end{aligned}
$$

and

$$
\begin{aligned}
& {\left[\frac{\hbar \boldsymbol{K}}{m} \cdot \nabla+\mathcal{E}\right] \bar{\rho}_{\alpha \beta}\left(\boldsymbol{R}, \boldsymbol{K} \mid \boldsymbol{r}_{1}\right)+\frac{2}{\hbar} \sum_{q}\left[\mathfrak{I}\left\{\int d^{3} R^{\prime} \tilde{u}_{a}\left(\boldsymbol{R}^{\prime}-\boldsymbol{r}_{1}\right) \mathrm{e}^{i \boldsymbol{q} \cdot\left(\boldsymbol{R}-\boldsymbol{R}^{\prime}\right)}\right\} \bar{\rho}_{\alpha \beta}\left(\boldsymbol{R}, \boldsymbol{K}+\frac{1}{2} \boldsymbol{q} \mid \boldsymbol{r}_{1}\right)\right.} \\
& \left.-\frac{b_{\mathrm{SO}}}{2} \mathfrak{I}\left\{\int d^{3} R^{\prime} \tilde{u}_{a}\left(\boldsymbol{R}^{\prime}-\boldsymbol{r}_{1}\right) \mathrm{e}^{i \boldsymbol{q} \cdot\left(\boldsymbol{R}-\boldsymbol{R}^{\prime}\right)} \kappa \cdot \sigma_{\beta \alpha}\right\} \bar{\rho}_{\alpha \alpha}\left(\boldsymbol{R}, \boldsymbol{K}+\frac{1}{2} \boldsymbol{q} \mid \boldsymbol{r}_{1}\right)\right] \\
& +\sum_{\boldsymbol{K}^{\prime}}\left\{T_{0}\left(\boldsymbol{K}, \boldsymbol{K}^{\prime}\right)\left[\bar{\rho}_{\alpha \beta}\left(\boldsymbol{R}, \boldsymbol{K} \mid \boldsymbol{r}_{1}\right)-\bar{\rho}_{\alpha \beta}\left(\boldsymbol{R}, \boldsymbol{K}^{\prime} \mid \boldsymbol{r}_{1}\right)\right]-T_{\mathrm{SO}}\left(\boldsymbol{K}, \boldsymbol{K}^{\prime}\right)\left[\bar{\rho}_{\alpha \alpha}\left(\boldsymbol{R}, \boldsymbol{K} \mid \boldsymbol{r}_{1}\right)-\bar{\rho}_{\alpha \alpha}\left(\boldsymbol{R}, \boldsymbol{K}^{\prime} \mid \boldsymbol{r}_{1}\right)\right]\right\}=0
\end{aligned}
$$

The approximate nature of (30) and (31) arises from $T\left(\boldsymbol{K}, \boldsymbol{K}^{\prime}\right)$ and $\gamma(\boldsymbol{K})$ not being modified in the presence of the potential fixed at $\boldsymbol{r}_{1}$. Here $\tilde{u}_{a}\left(\boldsymbol{R}^{\prime}-\boldsymbol{r}_{2}\right)$ is a modification of average potential and is different from the single ionic potential except in a totally random system. This potential is given by the formula

$$
\tilde{u}_{a}\left(\boldsymbol{R}^{\prime}-\boldsymbol{r}_{1}\right)=u_{a}\left(\boldsymbol{R}^{\prime}-\boldsymbol{r}_{1}\right)+\frac{N-1}{\Omega} \int d^{3} r_{2}\left[p\left(\boldsymbol{r}_{2} \mid \boldsymbol{r}_{1}\right)-1\right] u_{a}\left(\boldsymbol{R}^{\prime}-\boldsymbol{r}_{2}\right) .
$$

We can now interpret Eqs. (30) and (31) as transport equations for the effective medium, described by $T=T_{0}+T_{\text {so }}$, where $T_{\text {so }}$ is the generalized scattering kernel for spin-orbit interaction. We can eliminate $\bar{\rho}_{s^{\prime} s}\left(\boldsymbol{R}, \boldsymbol{K} \mid \boldsymbol{r}_{1}\right)$ from the Eqs. (31) and (32) by substituting $\bar{\rho}_{s^{\prime} s}\left(\boldsymbol{R}, \boldsymbol{K} \mid \boldsymbol{r}_{1}\right)=\bar{\rho}_{s^{\prime} s}(\boldsymbol{K})+\Delta \rho_{s^{\prime} s}\left(\boldsymbol{R}, \boldsymbol{K} \mid \boldsymbol{r}_{1}\right)$ 
and $\bar{\phi}\left(\boldsymbol{R}, \boldsymbol{K} \mid \boldsymbol{r}_{1}\right)=\bar{\phi}(\boldsymbol{K})+\Delta \bar{\phi}\left(\boldsymbol{R}, \boldsymbol{K} \mid \boldsymbol{r}_{1}\right)$ into these equations. As a result we obtain

$$
\begin{aligned}
& {\left[\frac{\hbar \boldsymbol{K}}{m} \cdot \nabla+\varepsilon\right] \Delta \bar{\rho}_{\alpha \alpha}\left(\boldsymbol{R}, \boldsymbol{K} \mid \boldsymbol{r}_{1}\right)+\frac{2}{\hbar} \sum_{q}\left[\mathfrak{I}\left\{\int d^{3} \boldsymbol{R}^{\prime} \tilde{u}_{a}\left(\boldsymbol{R}^{\prime}-\boldsymbol{r}_{1}\right) \mathrm{e}^{i \boldsymbol{q} \cdot\left(\boldsymbol{R}-\boldsymbol{R}^{\prime}\right)}\right\} \Delta \bar{\rho}_{\alpha \alpha}\left(\boldsymbol{R}, \boldsymbol{K}+\frac{1}{2} \boldsymbol{q} \mid \boldsymbol{r}_{1}\right)\right.} \\
& \left.-\frac{b_{\mathrm{SO}}}{2} \mathfrak{I}\left\{\int d^{3} \boldsymbol{R}^{\prime} \tilde{u}_{a}\left(\boldsymbol{R}^{\prime}-\boldsymbol{r}_{1}\right) \mathrm{e}^{i q \cdot\left(\boldsymbol{R}-\boldsymbol{R}^{\prime}\right)} \mathcal{K} \cdot \sigma_{\beta \alpha}\right\} \Delta \bar{\rho}_{\alpha \beta}\left(\boldsymbol{R}, \boldsymbol{K}+\frac{1}{2} \boldsymbol{q} \mid \boldsymbol{r}_{1}\right)\right] \\
& +\sum_{\boldsymbol{K}^{\prime}}\left\{T_{0}\left(\boldsymbol{K}, \boldsymbol{K}^{\prime}\right)\left[\Delta \bar{\rho}_{\alpha \alpha}\left(\boldsymbol{R}, \boldsymbol{K} \mid \boldsymbol{r}_{1}\right)-\Delta \bar{\rho}_{\alpha \alpha}\left(\boldsymbol{R}, \boldsymbol{K}^{\prime} \mid \boldsymbol{r}_{1}\right)\right]-T_{\mathrm{SO}}\left(\boldsymbol{K}, \boldsymbol{K}^{\prime}\right)\left[\Delta \bar{\rho}_{\alpha \beta}\left(\boldsymbol{R}, \boldsymbol{K} \mid \boldsymbol{r}_{1}\right)-\Delta \bar{\rho}_{\alpha \beta}\left(\boldsymbol{R}, \boldsymbol{K}^{\prime} \mid \boldsymbol{r}_{1}\right)\right]\right\} \\
& =-\frac{2}{\hbar} \sum_{q}\left[\mathfrak{J}\left\{\int d^{3} R^{\prime} \tilde{u}_{a}\left(\boldsymbol{R}^{\prime}-\boldsymbol{r}_{1}\right) \mathrm{e}^{i \boldsymbol{q} \cdot\left(\boldsymbol{R}-\boldsymbol{R}^{\prime}\right)}\right\} \bar{\rho}_{\alpha \alpha}\left(\boldsymbol{K}+\frac{1}{2} \boldsymbol{q}\right)\right. \\
& \left.-\frac{b_{\mathrm{SO}}}{2} \mathfrak{I}\left\{\int d^{3} R^{\prime} \tilde{u}_{a}\left(\boldsymbol{R}^{\prime}-\boldsymbol{r}_{1}\right) \mathrm{e}^{i \boldsymbol{q} \cdot\left(\boldsymbol{R}-\boldsymbol{R}^{\prime}\right)} \boldsymbol{\kappa} \cdot \sigma_{\beta \alpha}\right\} \bar{\rho}_{\alpha \beta}\left(\boldsymbol{K}+\frac{1}{2} \boldsymbol{q}\right)\right] \\
& -\frac{1}{3} e \boldsymbol{E} \cdot \boldsymbol{v}_{\alpha \alpha}(\boldsymbol{K})\left[1+\gamma_{0}(\boldsymbol{K})\right] \Delta \bar{\phi}\left(\boldsymbol{R}, \boldsymbol{K} \mid \boldsymbol{r}_{1}\right)
\end{aligned}
$$

and

$$
\begin{aligned}
& {\left[\frac{\hbar \boldsymbol{K}}{m} \cdot \nabla+\varepsilon\right] \Delta \bar{\rho}_{\alpha \beta}\left(\boldsymbol{R}, \boldsymbol{K} \mid \boldsymbol{r}_{1}\right)+\frac{2}{\hbar} \sum_{q}\left[\mathfrak{I}\left\{\int d^{3} R^{\prime} \tilde{u}_{a}\left(\boldsymbol{R}^{\prime}-\boldsymbol{r}_{1}\right) \mathrm{e}^{i \boldsymbol{q} \cdot\left(\boldsymbol{R}-\boldsymbol{R}^{\prime}\right)}\right\} \Delta \bar{\rho}_{\alpha \alpha}\left(\boldsymbol{R}, \boldsymbol{K}+\frac{1}{2} \boldsymbol{q} \mid \boldsymbol{r}_{1}\right)\right.} \\
& \left.-\frac{b_{\mathrm{SO}}}{2} \mathfrak{I}\left\{\int d^{3} R^{\prime} \tilde{u}_{a}\left(\boldsymbol{R}^{\prime}-\boldsymbol{r}_{1}\right) \mathrm{e}^{i \boldsymbol{q} \cdot\left(\boldsymbol{R}-\boldsymbol{R}^{\prime}\right)} \boldsymbol{\kappa} \cdot \sigma_{\alpha \beta}\right\} \Delta \bar{\rho}_{\alpha \alpha}\left(\boldsymbol{R}, \boldsymbol{K}+\frac{1}{2} \boldsymbol{q} \mid \boldsymbol{r}_{1}\right)\right] \\
& +\sum_{K^{\prime}}\left\{T_{0}\left(\boldsymbol{K}, \boldsymbol{K}^{\prime}\right)\left[\Delta \bar{\rho}_{\alpha \beta}\left(\boldsymbol{R}, \boldsymbol{K} \mid \boldsymbol{r}_{1}\right)-\Delta \bar{\rho}_{\alpha \beta}\left(\boldsymbol{R}, \boldsymbol{K}^{\prime} \mid \boldsymbol{R}_{1}\right)\right]-T_{\mathrm{SO}}\left(\boldsymbol{K}, \boldsymbol{K}^{\prime}\right)\left[\Delta \bar{\rho}_{\alpha \alpha}\left(\boldsymbol{R}, \boldsymbol{K} \mid \boldsymbol{r}_{1}\right)-\Delta \bar{\rho}_{\alpha \alpha}\left(\boldsymbol{R}, \boldsymbol{K}^{\prime} \mid \boldsymbol{r}_{1}\right)\right]\right\} \\
& =-\frac{2}{\hbar} \sum_{q}\left[\mathfrak{I}\left\{\int d^{3} R^{\prime} \tilde{u}_{a}\left(\boldsymbol{R}^{\prime}-\boldsymbol{r}_{1}\right) \mathrm{e}^{i \boldsymbol{q} \cdot\left(\boldsymbol{R}-\boldsymbol{R}^{\prime}\right)}\right\} \bar{\rho}_{\alpha \beta}\left(\boldsymbol{K}+\frac{1}{2} \boldsymbol{q}\right)\right. \\
& \left.-\frac{b_{\mathrm{SO}}}{2} \mathfrak{I}\left\{\int d^{3} R^{\prime} \tilde{u}_{a}\left(\boldsymbol{R}^{\prime}-\boldsymbol{r}_{1}\right) \mathrm{e}^{i \boldsymbol{q} \cdot\left(\boldsymbol{R}-\boldsymbol{R}^{\prime}\right)} \boldsymbol{\kappa} \cdot \sigma_{\alpha \beta}\right\} \bar{\rho}_{\alpha \alpha}\left(\boldsymbol{K}+\frac{1}{2} \boldsymbol{q}\right)\right] .
\end{aligned}
$$

These closed equations are extremely complex but this is the nature of the problem and we will now solve these equations approximately. It is very important to note that the operators on both sides of these equations are the same. In line with $[26,46]$ the terms involving the fixed potential on the left-hand side are omitted as it is reasonable for weak potentials, namely those which do not have resonant scattering properties like transition metals. In addition $T_{\mathrm{so}}\left(\boldsymbol{K}, \boldsymbol{K}^{\prime}\right)$ is omitted on the left-hand side as it should be small in comparison with $T_{0}\left(\boldsymbol{K}, \boldsymbol{K}^{\prime}\right)$. The complications of including $d$-bands and $d$-band conduction in an analytic fashion are extreme and would simply confuse the issues about spin-orbit interaction in this work. The solutions of (33) and (34) have the forms

$$
\begin{aligned}
\Delta \bar{\rho}_{\alpha \alpha}\left(\boldsymbol{R}, \boldsymbol{K} \mid \boldsymbol{r}_{1}\right) \approx & \sum_{\boldsymbol{K}^{\prime \prime}} \int d^{3} R^{\prime \prime} G\left(\boldsymbol{R}, \boldsymbol{R}^{\prime \prime}, \boldsymbol{K}, \boldsymbol{K}^{\prime \prime}\right)\left\{-\frac{2}{\hbar} \frac{1}{\Omega} \sum_{q} \mathfrak{I}\left\{\int d^{3} R^{\prime} \tilde{u}_{a}\left(\boldsymbol{R}^{\prime}-\boldsymbol{r}_{1}\right) \mathrm{e}^{i q \cdot\left(\boldsymbol{R}^{\prime \prime}-\boldsymbol{R}^{\prime}\right)}\right\} \bar{\rho}_{\alpha \alpha}\left(\boldsymbol{K}^{\prime \prime}+\frac{1}{2} \boldsymbol{q}\right)\right. \\
& \left.+\frac{b_{\mathrm{SO}}}{2} \mathfrak{I}\left\{\int d^{3} R^{\prime} \tilde{u}_{a}\left(\boldsymbol{R}^{\prime}-\boldsymbol{r}_{1}\right) \mathrm{e}^{i q \cdot\left(\boldsymbol{R}^{\prime \prime}-\boldsymbol{R}^{\prime}\right)} \boldsymbol{\kappa} \cdot \sigma_{\beta \alpha}\right\} \bar{\rho}_{\alpha \beta}\left(\boldsymbol{K}^{\prime \prime}+\frac{1}{2} \boldsymbol{q}\right)\right\} \\
& -\sum_{\boldsymbol{K}^{\prime \prime}} \int d^{3} R^{\prime \prime} G\left(\boldsymbol{R}, \boldsymbol{R}^{\prime \prime}, \boldsymbol{K}, \boldsymbol{K}^{\prime \prime}\right)\left[\frac{1}{3} e \boldsymbol{E} \cdot \boldsymbol{v}_{\alpha \alpha}\left(\boldsymbol{K}^{\prime \prime}\right)\left[1+\gamma_{0}\left(\boldsymbol{K}^{\prime \prime}\right)\right] \Delta \bar{\phi}\left(\boldsymbol{R}^{\prime \prime}, \boldsymbol{K}^{\prime \prime} \mid \boldsymbol{r}_{1}\right)\right]
\end{aligned}
$$


and

$$
\begin{aligned}
\Delta \bar{\rho}_{\alpha \beta}\left(\boldsymbol{R}, \boldsymbol{K} \mid \boldsymbol{r}_{1}\right) \approx \sum_{\boldsymbol{K}^{\prime \prime}} \int d^{3} R^{\prime \prime} G\left(\boldsymbol{R}, \boldsymbol{R}^{\prime \prime}, \boldsymbol{K}, \boldsymbol{K}^{\prime \prime}\right)\{ & -\frac{2}{\hbar} \frac{1}{\Omega} \sum_{q} \mathfrak{I}\left\{\int d^{3} R^{\prime} \tilde{u}_{a}\left(\boldsymbol{R}^{\prime}-\boldsymbol{r}_{1}\right) \mathrm{e}^{i \boldsymbol{q} \cdot\left(\boldsymbol{R}^{\prime \prime}-\boldsymbol{R}^{\prime}\right)}\right\} \bar{\rho}_{\alpha \beta}\left(\boldsymbol{K}^{\prime \prime}+\frac{1}{2} \boldsymbol{q}\right) \\
& \left.+\frac{b_{\mathrm{SO}}}{2} \mathfrak{I}\left\{\int d^{3} R^{\prime} \tilde{u}_{a}\left(\boldsymbol{R}^{\prime}-\boldsymbol{r}_{1}\right) \mathrm{e}^{i \boldsymbol{q} \cdot\left(\boldsymbol{R}^{\prime \prime}-\boldsymbol{R}^{\prime}\right)} \boldsymbol{\kappa} \cdot \sigma_{\alpha \beta}\right\} \bar{\rho}_{\alpha \alpha}\left(\boldsymbol{K}^{\prime \prime}+\frac{1}{2} \boldsymbol{q}\right)\right\} .
\end{aligned}
$$

These results can be substituted into (30) and (31), and the resulting equations compared directly to (16) and (17). We then obtain the self-consistent equations for $T\left(\boldsymbol{K}, \boldsymbol{K}^{\prime}\right)$ and $\gamma_{0}(\boldsymbol{K})$ as follows

$$
\begin{aligned}
T\left(\boldsymbol{K}, \boldsymbol{K}^{\prime}\right) \approx & \left(\frac{2}{\hbar}\right)^{2}\left(\frac{N}{\Omega}\right)^{2} \sum_{q} \sum_{q^{\prime}} \int d^{3} R\left[\mathfrak{I}\left\{\int d^{3} R^{\prime} d^{3} r_{1} p\left(\boldsymbol{r}_{1}\right) u_{a}\left(\boldsymbol{R}^{\prime}-\boldsymbol{R}_{1}\right) \mathrm{e}^{i q \cdot\left(\boldsymbol{R}-\boldsymbol{R}^{\prime}\right)}\right\}\right. \\
& \times \int d^{3} R^{\prime \prime} G\left(\boldsymbol{R}, \boldsymbol{R}^{\prime \prime}, \boldsymbol{K}+\frac{1}{2} \boldsymbol{q}, \boldsymbol{K}^{\prime}-\frac{1}{2} \boldsymbol{q}^{\prime}\right) \mathfrak{I}\left\{\int d^{3} R^{\prime \prime \prime} \tilde{u}_{a}\left(\boldsymbol{R}^{\prime \prime \prime}-\boldsymbol{R}_{1}\right) \mathrm{e}^{i \boldsymbol{q} \cdot\left(\boldsymbol{R}^{\prime \prime}-\boldsymbol{R}^{\prime \prime \prime}\right)}\right\} \\
& +\left(\frac{b_{\mathrm{SO}}}{2}\right)^{2} \mathfrak{I}\left\{\int d^{3} R^{\prime} d^{3} r_{1} p\left(\boldsymbol{r}_{1}\right) u_{a}\left(\boldsymbol{R}^{\prime}-\boldsymbol{r}_{1}\right) \mathrm{e}^{i \boldsymbol{q} \cdot\left(\boldsymbol{R}-\boldsymbol{R}^{\prime}\right)} \kappa \cdot \sigma_{\alpha \beta}\right\} \\
& \left.\times \int d^{3} R^{\prime \prime} G\left(\boldsymbol{R}, \boldsymbol{R}^{\prime \prime}, \boldsymbol{K}+\frac{1}{2} \boldsymbol{q}, \boldsymbol{K}^{\prime}-\frac{1}{2} \boldsymbol{q}^{\prime}\right) \mathfrak{I}\left\{\int d^{3} R^{\prime \prime \prime} \tilde{u}_{a}\left(\boldsymbol{R}^{\prime \prime \prime}-\boldsymbol{R}_{1}\right) \mathrm{e}^{i \boldsymbol{q} \cdot\left(\boldsymbol{R}^{\prime \prime}-\boldsymbol{R}^{\prime \prime \prime}\right)} \kappa^{\prime} \cdot \sigma_{\beta \alpha}\right\}\right]
\end{aligned}
$$

and

$$
\begin{aligned}
\gamma_{0}(\boldsymbol{K})= & -\frac{2}{\hbar} \frac{N}{\Omega^{2}} \sum_{K^{\prime}} \sum_{q^{\prime}} \int d^{3} R \mathfrak{I}\left\{\int d^{3} R^{\prime} d^{3} r_{1} p_{1}\left(\boldsymbol{r}_{1}\right) u_{a}\left(\boldsymbol{R}^{\prime}-\boldsymbol{r}_{1}\right) \mathrm{e}^{i \boldsymbol{q} \cdot\left(\boldsymbol{R}-\boldsymbol{R}^{\prime}\right)}\right\} \\
& \times \int d^{3} R^{\prime \prime} G\left(\boldsymbol{R}, \boldsymbol{R}^{\prime \prime}, \boldsymbol{K}+\frac{1}{2} \boldsymbol{q}, \boldsymbol{K}^{\prime}\right) \frac{v_{\alpha \alpha}\left(K_{x}^{\prime}\right)}{v_{\alpha \alpha}\left(K_{x}\right)} \frac{\bar{\phi}\left(\boldsymbol{R}^{\prime \prime}, \boldsymbol{K}^{\prime} \mid \boldsymbol{r}_{1}\right)-\bar{\phi}\left(\boldsymbol{K}^{\prime}\right)}{\bar{\phi}(\boldsymbol{K})}\left[1+\gamma_{0}\left(\boldsymbol{K}^{\prime}\right)\right] .
\end{aligned}
$$

The generalized scattering kernel consists of ordinary and spin-orbit scattering terms. Equation (16) together with (37) and (38) is a central result of this section. Because $G\left(\boldsymbol{R}, \boldsymbol{R}^{\prime}\right)$ only depends on $\boldsymbol{R}-\boldsymbol{R}^{\prime}$, due to neglecting $\tilde{u}_{a}$ on the left hand side of (34), the kernel $T\left(\boldsymbol{K}, \boldsymbol{K}^{\prime}\right)$ can be rewritten in the form

$$
\begin{aligned}
T\left(\boldsymbol{K}, \boldsymbol{K}^{\prime}\right) \approx & \frac{1}{\hbar^{2}} \frac{N}{\Omega^{2}} \sum_{\boldsymbol{Q}} u_{a}(\boldsymbol{Q}) \tilde{u}_{a}(-\boldsymbol{Q})\left(1-\delta_{K, \boldsymbol{K}^{\prime}}\right) \\
& \times\left[\left\{1+b_{\mathrm{SO}}^{2}\left[(\boldsymbol{K} \times \boldsymbol{Q}) \cdot \sigma_{\beta \alpha}\right]\left[\left(\boldsymbol{K}^{\prime} \times \boldsymbol{Q}\right) \cdot \sigma_{\alpha \beta}\right]\right\} G\left(\boldsymbol{Q}, \boldsymbol{K}-\frac{1}{2} \boldsymbol{Q}, \boldsymbol{K}^{\prime}+\frac{1}{2} \boldsymbol{Q}\right)\right. \\
& +\left\{1+b_{\mathrm{SO}}^{2}\left[(\boldsymbol{K} \times \boldsymbol{Q}) \cdot \sigma_{\alpha \beta}\right]\left[\left(\boldsymbol{K}^{\prime} \times \boldsymbol{Q}\right) \cdot \sigma_{\beta \alpha}\right]\right\} G\left(\boldsymbol{Q}, \boldsymbol{K}+\frac{1}{2} \boldsymbol{Q}, \boldsymbol{K}^{\prime}-\frac{1}{2} \boldsymbol{Q}\right) \\
& -\left\{1+b_{\mathrm{SO}}^{2}\left[(\boldsymbol{K} \times \boldsymbol{Q}) \cdot \sigma_{\beta \alpha}\right]\left[\left(\boldsymbol{K}^{\prime} \times \boldsymbol{Q}\right) \cdot \sigma_{\alpha \beta}\right]\right\} G\left(\boldsymbol{Q}, \boldsymbol{K}+\frac{1}{2} \boldsymbol{Q}, \boldsymbol{K}^{\prime}+\frac{1}{2} \boldsymbol{Q}\right) \\
& \left.-\left\{1+b_{\mathrm{SO}}^{2}\left[(\boldsymbol{K} \times \boldsymbol{Q}) \cdot \sigma_{\alpha \beta}\right]\left[\left(\boldsymbol{K}^{\prime} \times \boldsymbol{Q}\right) \cdot \sigma_{\beta \alpha}\right]\right\} G\left(\boldsymbol{Q}, \boldsymbol{K}-\frac{1}{2} \boldsymbol{Q}, \boldsymbol{K}^{\prime}-\frac{1}{2} \boldsymbol{Q}\right)\right] .
\end{aligned}
$$

Even at this stage the general form for $G$ is not known nor is it known in closed form except for the simplest type of Boltzmann equation with isotropic elastic scattering. However the Fourier transform of the exact Green function which is involved in (39) can be expressed in the form of a multiple scattering series representing successive scattering events, describing the distribution resulting from a point source of particles

$$
G\left(\boldsymbol{Q}, \boldsymbol{K}, \boldsymbol{K}^{\prime}\right)=G_{0}(\boldsymbol{Q}, \boldsymbol{K}) \delta_{\boldsymbol{K}, \boldsymbol{K}^{\prime}}+G_{0}(\boldsymbol{Q}, \boldsymbol{K}) T\left(\boldsymbol{K}, \boldsymbol{K}^{\prime}\right) G_{0}\left(\boldsymbol{Q}, \boldsymbol{K}^{\prime}\right)+\ldots,
$$


where $G_{0}(\boldsymbol{Q}, \boldsymbol{K})$ is essentially the Wigner Green function for free electrons but attenuated in the medium and is defined by

$$
G_{0}(\boldsymbol{Q}, \boldsymbol{K})=\left[i \frac{\hbar \boldsymbol{K}}{m} \cdot \boldsymbol{Q}+\tau^{-1}(\boldsymbol{K})\right]^{-1} .
$$

It perhaps should be noted that a point source with well defined momentum is not allowed due to the Heisenberg uncertainty principle but it is allowed mathematically within the Wigner formulation. As has been stated the series (40) can be summed when $T\left(\boldsymbol{K}, \boldsymbol{K}^{\prime}\right)$ is isotropic and $|\boldsymbol{K}|=\left|\boldsymbol{K}^{\prime}\right|$ but this summation is not possible in a general way. If $|\boldsymbol{K}|=\left|\boldsymbol{K}^{\prime}\right|$ then the result for small $Q$ (large $\boldsymbol{R}$ ) can be obtained as this corresponds to the solution of the diffusion equation and $G$ has a diffusion singularity, namely $G \propto|Q|^{-2}$. However QI is obtained from the first two terms in Eq. (40) whereas the long range behaviour can be used to discuss localization. The function $\tau^{-1}(\boldsymbol{K})$ is the relaxation time which is defined by the following formula: $\tau^{-1}(\boldsymbol{K})=\sum_{\boldsymbol{K}^{\prime}} T\left(\boldsymbol{K}, \boldsymbol{K}^{\prime}\right)$ [26]. As has been mentioned before the skew scattering is zero at this level of approximation. If we substitute the first term of series (40) into (39), and next we calculate the sum of (39) over $\boldsymbol{K}^{\prime}$, we obtain a self-consistent equation for the relaxation time, namely

$$
\tau^{-1}(\boldsymbol{K})=\frac{2 \pi}{\hbar} \frac{N}{\Omega^{2}} \sum_{\boldsymbol{K}^{\prime}} \frac{u_{a}\left(\boldsymbol{K}^{\prime}-\boldsymbol{K}\right) \tilde{u}_{a}\left(\boldsymbol{K}-\boldsymbol{K}^{\prime}\right)\left[1+b_{\mathrm{SO}}^{2}\left|\left(\boldsymbol{K} \times \boldsymbol{K}^{\prime}\right) \sigma_{\alpha \beta}\right|^{2}\right] \hbar \tau^{-1}\left(\frac{\boldsymbol{K}+\boldsymbol{K}^{\prime}}{2}\right)}{\pi\left\{\left[\frac{\hbar^{2}}{2 m}\left(\boldsymbol{K}^{\prime 2}-\boldsymbol{K}^{2}\right)\right]^{2}+\left[\hbar \tau^{-1}\left(\frac{\boldsymbol{K}+\boldsymbol{K}^{\prime}}{2}\right)\right]^{2}\right\}} .
$$

The inclusion of a finite life time under the summation sign in (42) is not really an important issue in this work.

The comments made earlier about the use of pseudopotentials to calculate spin-orbit effects can now be clarified. We note that the normal scattering is essentially multiplied by $b_{\mathrm{SO}}^{2} K^{4}$ where $K$ is the value at the Fermi energy. Since $b_{\mathrm{SO}}^{2}=14.9 \times 10^{-52} \mathrm{~m}^{4}$ and $K=1.2 \times 10^{10} \mathrm{~m}^{-1}$ it can be seen that the spin-orbit scattering appears smaller by a factor of about $10^{10}$ compared with the normal scattering. This is not true [51] and this result stems from the fact that $u_{a}(q)$ is the result of taking the matrix element of an atomic like potential between OPW states. The use of Eq. (1) and the appearance of the Fourier transform of $u_{a}(q)$ corresponds to taking the derivative of a weak potential in real space. As has been clearly shown by Liu [21] the matrix elements of the spin-orbit operator for the true potential using OPW's are totally dominated by the core states and strongly dependent on the atomic number. Hence although we will retain the form (42) for simplicity we must recognize that the pseudopotential terms must be hugely enhanced in actual estimates of the spin-orbit scattering. In the calculations of Brown and Morgan [51] for $\mathrm{Au}$ and $\mathrm{Cu}$ impurities in $\mathrm{Ag}$ the scattering from $\mathrm{Au}$ atoms is characterized by phase shifts for $l=0,1,2$ and the magnitude of the spin-orbit scattering by an effective phase shift which is only significant for $l=1$ but comparable with the normal potential phase shifts. In fact for Au impurities the resistance is dominated by the mass-velocity and Darwin relativistic corrections to the scattering but the point is that the normal potential scattering and spin-orbit scattering is comparable. For $\mathrm{Cu}$ impurities the spin-orbit scattering is rather smaller due to the strong $Z$ dependence of spin-orbit scattering.

\section{Quantum interference}

To investigate quantum interference effects it is convenient to separate the generalized scattering kernel into three parts, namely

$$
T\left(\boldsymbol{K}, \boldsymbol{K}^{\prime}\right)=T_{1}\left(\boldsymbol{K}, \boldsymbol{K}^{\prime}\right)+T_{2}\left(\boldsymbol{K}, \boldsymbol{K}^{\prime}\right)+T_{3}\left(\boldsymbol{K}, \boldsymbol{K}^{\prime}\right),
$$

where $T_{1}\left(\boldsymbol{K}, \boldsymbol{K}^{\prime}\right)$ is produced by the first term in (40). 
These have been chosen so that they represent, respectively, the normal Boltzmann equation scattering obtained by perturbation theory with lifetime broadening, the multiple scattering peaked in the backward direction $T_{2}\left(\boldsymbol{K}, \boldsymbol{K}^{\prime}\right)$, and the multiple scattering peaked in the forward direction $T_{3}\left(\boldsymbol{K}, \boldsymbol{K}^{\prime}\right)$. The characteristic property of this splitting is that $T_{2}\left(\boldsymbol{K}, \boldsymbol{K}^{\prime}\right)+T_{3}\left(\boldsymbol{K}, \boldsymbol{K}^{\prime}\right)$ summed over $\boldsymbol{K}^{\prime}$ is equal to zero, but if $T_{2}\left(\boldsymbol{K}, \boldsymbol{K}^{\prime}\right)+T_{3}\left(\boldsymbol{K}, \boldsymbol{K}^{\prime}\right)$ is summed with the factor $\left(1-\cos \theta_{\boldsymbol{K}, \boldsymbol{K}^{\prime}}\right)$ weighting against forward scattering then the cancellation does not occur. As it was shown in [26], the QI effects are represented by $T_{2}\left(\boldsymbol{K}, \boldsymbol{K}^{\prime}\right)$. When spin-orbit interaction is included $T_{2}\left(\boldsymbol{K}, \boldsymbol{K}^{\prime}\right)$ takes the form

$$
\begin{aligned}
T_{2}\left(\boldsymbol{K}, \boldsymbol{K}^{\prime}\right)= & \frac{1}{\hbar^{2}} \frac{N}{\Omega^{2}} \sum_{\boldsymbol{Q}} u_{a}(\boldsymbol{Q}) \tilde{u}_{a}(-\boldsymbol{Q}) \\
& \times\left[\left(1-\delta_{\boldsymbol{K}-\boldsymbol{K}^{\prime}, \boldsymbol{Q}}\right)\left\{1+b_{\mathrm{SO}}^{2}\left[(\boldsymbol{K} \times \boldsymbol{Q}) \cdot \sigma_{\beta \alpha}\right]\left[\left(\boldsymbol{K}^{\prime} \times \boldsymbol{Q}\right) \cdot \sigma_{\alpha \beta}\right]\right\} G\left(\boldsymbol{Q}, \boldsymbol{K}-\frac{1}{2} \boldsymbol{Q}, \boldsymbol{K}^{\prime}+\frac{1}{2} \boldsymbol{Q}\right)\right. \\
& \left.+\left(1-\delta_{\boldsymbol{K}^{\prime}-\boldsymbol{K}, \boldsymbol{Q}}\right)\left\{1+b_{\mathrm{SO}}^{2}\left[(\boldsymbol{K} \times \boldsymbol{Q}) \cdot \sigma_{\alpha \beta}\right]\left[\left(\boldsymbol{K}^{\prime} \times \boldsymbol{Q}\right) \cdot \sigma_{\beta \alpha}\right]\right\} G\left(\boldsymbol{Q}, \boldsymbol{K}+\frac{1}{2} \boldsymbol{Q}, \boldsymbol{K}^{\prime}-\frac{1}{2} \boldsymbol{Q}\right)\right] .
\end{aligned}
$$

This can be converted into a self-consistent equation using the Green function defined in (40) and neglecting $T_{3}\left(\boldsymbol{K}, \boldsymbol{K}^{\prime}\right)$, which was proposed in [26], but its structure is a little different, because the spinorbit interaction is included. A generalized form of (40) corresponding to an interpolation between small $\boldsymbol{Q}$ (large $\boldsymbol{R}$ ) and large $\boldsymbol{Q}$ (small $\boldsymbol{R}$ ) is [26]

$$
G\left(\boldsymbol{Q}, \boldsymbol{K}, \boldsymbol{K}^{\prime}\right) \approx \frac{\delta_{\boldsymbol{K}, \boldsymbol{K}^{\prime}}}{i \frac{\hbar \boldsymbol{K}}{m} \cdot \boldsymbol{Q}+\tau^{-1}(\boldsymbol{K})}+\frac{T\left(\boldsymbol{K}, \boldsymbol{K}^{\prime}\right)+3 \bar{T}_{1}\left(\boldsymbol{K}, \boldsymbol{K}^{\prime}\right) \tau^{-1}(\boldsymbol{K}) \tau_{t r}^{-1}(\boldsymbol{K})\left(\frac{m}{\hbar K Q}\right)^{2}}{\left[i \frac{\hbar \boldsymbol{K}}{m} \cdot \boldsymbol{Q}+\tau^{-1}(\boldsymbol{K})\right]\left[i \frac{\hbar \boldsymbol{K}^{\prime}}{m} \cdot \boldsymbol{Q}+\tau^{-1}\left(\boldsymbol{K}^{\prime}\right)\right]},
$$

where $\bar{T}_{1}\left(\boldsymbol{K}, \boldsymbol{K}^{\prime}\right)$ is defined as an average of $T_{1}\left(\boldsymbol{K}, \boldsymbol{K}^{\prime}\right)$ over angles and $\tau_{t r}$ is a transport lifetime. However the term involving $\bar{T}_{1}\left(\boldsymbol{K}, \boldsymbol{K}^{\prime}\right)$ is not important in the context of quantum interference and will be omitted although it is important in estimating when electrons become localized. Substituting (40) into (44) yields the equation for $T_{2}\left(K, K^{\prime}\right)$ in the form

$$
T_{2}\left(\boldsymbol{K}, \boldsymbol{K}^{\prime}\right) \approx T_{1}\left(\boldsymbol{K}, \boldsymbol{K}^{\prime}\right)+T_{2}\left(\boldsymbol{K}, \boldsymbol{K}^{\prime}\right) R
$$

where the form (46) results from the fact that $T_{2}\left(\boldsymbol{K}, \boldsymbol{K}^{\prime}\right)$ turns out to be strongly peaked in the backward direction [26]. $R$ is defined by

$$
R=\frac{2}{\hbar^{2}} \frac{N}{\Omega^{2}} \Re\left\{\sum_{Q} Z(\boldsymbol{Q})\right\},
$$

where $Z(Q)$ is given by the formula

$$
Z(\boldsymbol{Q})=\frac{u_{a}(\boldsymbol{Q}) \tilde{u}_{a}(-\boldsymbol{Q})\left\{1+b_{\mathrm{SO}}^{2}\left[(\boldsymbol{K} \times \boldsymbol{Q}) \cdot \sigma_{\beta \alpha}\right]\left[\left(\boldsymbol{K}^{\prime} \times \boldsymbol{Q}\right) \cdot \sigma_{\alpha \beta}\right]\right\}}{\left[i \frac{\hbar\left(\boldsymbol{K}-\frac{1}{2} \boldsymbol{Q}\right)}{m} \boldsymbol{Q}+\tau^{-1}\left(\boldsymbol{K}-\frac{1}{2} \boldsymbol{Q}\right)\right]\left[i \frac{\hbar\left(\boldsymbol{K}^{\prime}+\frac{1}{2} \boldsymbol{Q}\right)}{m} \boldsymbol{Q}+\tau^{-1}\left(\boldsymbol{K}^{\prime}+\frac{1}{2} \boldsymbol{Q}\right)\right]} .
$$

The statement that $T_{2}\left(\boldsymbol{K}, \boldsymbol{K}^{\prime}\right)$ is strongly peaked in the backward direction stems from the fact the equation for $T_{2}\left(\boldsymbol{K}, \boldsymbol{K}^{\prime}\right)$ which is simplified in (46) corresponds to summing the maximally crossed diagrams for the vertex part when further simplifying assumptions are made about the scattering potential. We will first consider the modification of the normal Boltzmann scattering which comes from the quantum interference effects and specifically the effects of spin-orbit interaction. After the change of variables $\boldsymbol{Q}=\boldsymbol{K}-\boldsymbol{q}$ and $\boldsymbol{K}^{\prime}=-\boldsymbol{K}+\Delta \boldsymbol{K}$, where $\Delta \boldsymbol{K}$ goes to zero for the backward direction, and using the 
form for small $Q$ [26], Eq. (44) can be rewritten in the form

$$
T_{2}\left(\boldsymbol{K}, \boldsymbol{K}^{\prime}\right) \approx \frac{T_{1}\left(\boldsymbol{K}, \boldsymbol{K}^{\prime}\right)+T_{2}^{\text {as }}\left(\boldsymbol{K}, \boldsymbol{K}^{\prime}\right)}{A+B(\Delta \boldsymbol{K})^{2}},
$$

where $T_{2}^{\text {as }}\left(\boldsymbol{K}, \boldsymbol{K}^{\prime}\right)$ comes from integrating the term involving the small $\boldsymbol{Q}$ behaviour. When $A$ is set equal to zero, as is usually assumed, there is singularity of the form $|\Delta \boldsymbol{K}|^{-2}$. When dephasing due to atomic motion is included then this removes the singularity. The dephasing time is not the electron-phonon relaxation time but has been calculated by Bruce and Morgan [46] using similar methods to this paper but the ensemble averages in this paper are replaced by time averages over the atomic motion. Taking $A$ to be zero corresponds to neglecting the dependence of $\tau^{-1}$ on $\boldsymbol{K}$ in (42) which has been the procedure in all papers that we know of dealing with quantum interference. The outcome of $A$ not being exactly equal to zero are profound because this removes or changes the nature of the singularity and modifies the effect of dephasing due to various sources but this is another issue which requires extensive discussion. However in this paper we find that the spin-orbit interaction modifies the magnitude of the singularity instead of removing it so that the behaviour of $A$ is not so significant. In (49) $B=\left[(\hbar \boldsymbol{K} / m)^{2} \tau^{2}\right] / 3$, and $\tau^{-1}=\tau_{\mathrm{el}}^{-1}+\tau_{\mathrm{SO}}^{-1} \approx \tau_{\mathrm{el}}^{-1}$, where $\tau_{\mathrm{el}}$ is the elastic scattering time.

If we consider (48) and $\boldsymbol{K}^{\prime}=-\boldsymbol{K}$ then the inclusion of spin-orbit scattering does not affect the behaviour of $A$. However we can see that when we expand the denominator in powers of $\Delta \boldsymbol{K}$ there are terms which are linear in $\Delta \boldsymbol{K}$ but multiply linear terms arising from $\Delta \boldsymbol{K} \times \boldsymbol{Q}$. These terms can have a large effect even though the spin-orbit coupling is weak due to the fact that the denominator also has terms of the order of $\Delta \boldsymbol{K}$.

We replace the summation in (47) by integration and we obtain

$$
R=\frac{1}{2 \pi^{2} \hbar^{2}} \frac{N}{\Omega} \Re\left\{\int d^{3} q Z(\boldsymbol{K}-\boldsymbol{q})\right\} .
$$

We next expand the denominator of (50) into power series of $\Delta \boldsymbol{K}$ to the second order of $\Delta \boldsymbol{K}$. After multiplication of this expansion by the numerator, which is linear in $\Delta \boldsymbol{K}$, we obtain six integrals but only three of them are important in our case. The first one is

$$
I_{1}=\frac{1}{(2 \pi \hbar)^{2}} \frac{N}{\Omega} \frac{1}{\pi} \int d^{3} q \frac{u_{a}(\boldsymbol{K}-\boldsymbol{q}) \tilde{u}_{a}(\boldsymbol{q}-\boldsymbol{K})\left\{1+b_{\mathrm{SO}}^{2}\left|(\boldsymbol{K} \times \boldsymbol{q}) \sigma_{\alpha \beta}\right|^{2}\right\}}{\frac{1}{4}\left(\frac{\hbar}{m}\right)^{2}\left(K^{2}-q^{2}\right)^{2}+\tau^{-2}} .
$$

The value of this integral is approximately equal to unity as has been discusssed and is usually assumed to be so in most works. The next important integral has the form

$$
\begin{aligned}
I_{4}= & \frac{1}{(2 \pi \hbar)^{2}} \frac{N}{\Omega} \frac{1}{\pi} \int d^{3} q \frac{u_{a}(\boldsymbol{K}-\boldsymbol{q}) \tilde{u}_{a}(\boldsymbol{q}-\boldsymbol{K})\left\{1+b_{\mathrm{so}}^{2}\left|(\boldsymbol{K} \times \boldsymbol{q}) \cdot \sigma_{\alpha \beta}\right|^{2}\right\}}{\frac{1}{4}\left(\frac{\hbar}{m}\right)^{2}\left(K^{2}-q^{2}\right)^{2}+\tau^{-2}} \\
& \times \Re\left\{\frac{\left[\frac{1}{2}\left(\frac{\hbar}{m}\right)\left(K^{2}-q^{2}\right)-i\left(\frac{\hbar}{m}\right) \tau^{-1}\right]^{2}}{\left[\frac{1}{4}\left(\frac{\hbar}{m}\right)^{2}\left(K^{2}-q^{2}\right)^{2}+\tau^{-1}\right]^{2}}\right\}(\Delta \boldsymbol{K} \cdot \boldsymbol{q})^{2} .
\end{aligned}
$$

This integral is like that in our previous work on the ' $2 k_{F}{ }^{\prime}$-scattering [26] and can be approximated by

$$
I_{4} \approx-\frac{1}{3}\left(\frac{\hbar}{m}\right)^{2} K^{2} \tau^{2}|\Delta K|^{2} .
$$


The calculation of

$$
\begin{aligned}
I_{5}= & \frac{1}{(2 \pi \hbar)^{2}} \frac{N}{\Omega} \frac{b_{\mathrm{SO}}^{2}}{\pi} \int d^{3} q \frac{u_{a}(\boldsymbol{K}-\boldsymbol{q}) \tilde{u}_{a}(\boldsymbol{q}-\boldsymbol{K})\left[(\boldsymbol{K} \times \boldsymbol{q}) \cdot \sigma_{\beta \alpha}\right]\left[(\Delta \boldsymbol{K} \times \boldsymbol{K}) \cdot \sigma_{\alpha \beta}\right]}{\frac{1}{4}\left(\frac{\hbar}{m}\right)^{2}\left(K^{2}-q^{2}\right)^{2}+\tau^{-2}} \\
& \times \Re\left\{\frac{\left.\frac{\frac{1}{2}\left(\frac{\hbar}{m}\right)^{2}\left(K^{2}-q^{2}\right)-i\left(\frac{\hbar}{m}\right) \tau^{-1}}{\frac{1}{4}\left(\frac{\hbar}{m}\right)^{2}\left(K^{2}-q^{2}\right)^{2}+\tau^{-2}}\right\}(\Delta \boldsymbol{K} \cdot \boldsymbol{q})}{}\right.
\end{aligned}
$$

is a little bit more complicated but after some lengthy calculations we can approximate the value of $I_{5}$ by

$$
I_{5} \approx-\frac{1}{3} b_{\mathrm{SO}}^{2}\left(\frac{\hbar}{m}\right)^{2} K^{4} K_{0}^{2} \tau^{2}|\Delta \boldsymbol{K}|^{2},
$$

where $K_{0}^{2}=2 \sqrt{K^{4}+\left[2 m(\hbar \tau)^{-1}\right]^{2}}$. The sign of $I_{5}$ is negative because of the term $K^{2}-q^{2}$ in the numerator of the integrand. The constant $K_{0}^{2}$ results from an approximation made for the integration of the sharply peaked functions. It should be noted that many model calculations make much more extreme simplifying assumptions such as replacing the true potentials by a random potential.

Both integrals are proportional to $|\Delta \boldsymbol{K}|^{2}$. This is important point because they both contribute to the WL terms [52]. Finally Eq. (50) has the form $R \approx 1+I_{4}+I_{5}$. We substitute it into Eq. (46) and after some simple algebraic manipulation we obtain the equation for $T_{2}\left(\boldsymbol{K}, \boldsymbol{K}^{\prime}\right)$ and hence the inverse of relaxation time

$$
\begin{aligned}
\tau_{\text {tr }}^{-1}= & \sum_{\boldsymbol{K}^{\prime}} T_{1}\left(\boldsymbol{K}, \boldsymbol{K}^{\prime}\right)\left(1-\cos \theta_{\boldsymbol{K} K^{\prime}}\right) \\
& +\sum_{\boldsymbol{K}^{\prime}} T_{2}\left(\boldsymbol{K}, \boldsymbol{K}^{\prime}\right)\left(1-\cos \theta_{\boldsymbol{K} K^{\prime}}\right) .
\end{aligned}
$$

The first part gives the usual Boltzmann contribution, namely the inverse Faber-Ziman relaxation time $\tau_{\mathrm{FZ}}^{-1}$ whereas the second one gives the QI correction. The latter comes mainly from the backward scattering. Thus we can put $\cos \theta_{K K^{\prime}}=-1$ and then

$$
\tau_{\mathrm{tr}}^{-1}=\tau_{\mathrm{FZ}}^{-1}+2 \sum_{\boldsymbol{K}^{\prime}} T_{2}\left(\boldsymbol{K}, \boldsymbol{K}^{\prime}\right) .
$$

The QI enhancement of the electrical resistivity in the Faber-Ziman model when the spin-orbit scattering is neglected was considered by Howson et al. [53]. The inverse of the relaxation time obtained in this case will be denoted by $\left(\tau_{\mathrm{tr}}^{-1}\right)^{\prime}$. If we include the spin-orbit scattering, then the inverse of transport relaxation time is given by

$$
\left(\tau_{\mathrm{tr}}^{-1}\right)^{\prime \prime}=\tau_{\mathrm{FZ}}^{-1}-2 \sum_{K^{\prime}} \frac{T_{1}\left(\boldsymbol{K}, \boldsymbol{K}^{\prime}\right)}{I_{4}+I_{5}} .
$$

It should give rise to a decrease of total resistance because both $I_{4}$ and $I_{5}$ are negative. Thus the QI part is diminished due to the spin-orbit scattering but the singularity is not removed.

It is convenient to introduce the relative change of resistivity for comparison with experiment

$$
\frac{\Delta \rho}{\rho_{0}}=\frac{\left(\tau_{\mathrm{tr}}^{-1}\right)^{\prime \prime}-\left(\tau_{\mathrm{tr}}^{-1}\right)^{\prime}}{\left(\tau_{\mathrm{tr}}^{-1}\right)^{\prime}} .
$$

After some lengthy but straightforward calculations we obtain

$$
\frac{\Delta \rho}{\rho_{0}} \approx-b_{\mathrm{SO}}^{2} K^{2} K_{0}^{2} \frac{\frac{1}{2} \tau^{-1} X^{2} F_{\mathrm{MHS}}^{1 / 2}(X)}{\tau_{\mathrm{FZ}}^{-1}+\frac{1}{2} \tau^{-1} X^{2} F_{\mathrm{MHS}}^{1 / 2}(X)},
$$


where $X=\hbar /\left(\tau E_{\mathrm{F}}\right), E_{\mathrm{F}}$ is the Fermi energy and the function $F_{\mathrm{MHS}}^{1 / 2}(X)$ was defined in [53]. $F_{\mathrm{MHS}}^{1 / 2}(X)$ is always positive so that $\Delta \rho / \rho_{0}<0$. An important point to be emphasized is that the magnitude of $I_{5}$ is significant because it arises from the product of two terms linear in $\Delta \boldsymbol{K}$ one of which does not involve $b_{\text {so }}$.

The negative sign of the spin-orbit contribution to the resistivity has been explained in qualitative terms as follows [54]. In highly scattering media a conduction electron can go along a closed path and return to the starting point. Its wave function interferes here with another path which represents the electron going the same path but in the opposite direction. The movement of the electron along a closed path is equivalent to the rotation of the coordinate frame by $2 \pi$ so that the spinors describing the spin states of both electrons change their signs to opposite ones. If there are no other disturbing factors, for example phonons or magnetic fields which may change the phase of the wave functions, they interfere constructively. It gives rise to weak localization and causes an increase in the resistivity of highly disordered materials. If we now assume the existence of impurities of heavy ions which scatter electrons due to spin-orbit interaction the phase coherence is partly destroyed. Specifically, the spin parts of the electron wave functions are not the same. The localization of electrons is diminished and conduction properties of the material are enhanced. This gives rise to negative change in the resistivity as obtained in the Eq. (60). A similar effect was predicted by Hikami et al. [30]. According to their calculations the spin-orbit interaction produces a leading contribution to the $\beta$ function of a scaling theory $[55,56]$ of the opposite sign to that given by perturbation theory. This gives rise to the change in the behaviour of the conductance. It means that our theory is consistent with that of Hikami et al. and the physical interpretation.

\section{Conclusions}

Our intention was to formulate a quantitative theory describing the effect of the spin-orbit scattering on the electrical conductivity of disordered systems which would be an alternative to other approaches [55, 57]. Our final formula (60)) can be used for the explanation of experimental results for the resistivity of amorphous alloys of simple metals with a heavy metal component where the spin-orbit scattering is important. Sahnoune et al. [13] published their results of measurements of the resistivity for a series of amorphous alloys $\mathrm{Ca}_{70} \mathrm{Al}_{30-x} \mathrm{Au}_{x}$ which belong to this category, and explained them in terms of the Fukuyama and Hoshino theory [16]. We think that this theory is not suitable for this class of alloys because its range of validity is supposed to be when $k_{\mathrm{F}} l \gg 1$ which is not the case for these alloys and is one the reasons for our work.

Our theory is suitable both for weak $\left(k_{\mathrm{F}} l \gg 1\right)$ as well as strong $\left(k_{\mathrm{F}} l \approx 1\right)$ scattering range and predicts the negative change of resistivity in both cases in agreement with experiment. The quantitative calculations of the resistivity based on the formula (60) would need a good knowledge of structure factors and pseudopotentials for a specific materials. It will be a subject of future work.

Acknowledgement The authors thank Mr. Keith MacKenna for helpful discussions. The autors also thank the British Council and the Polish State Committee for Scientific Research for financial support.

\section{References}

[1] T. Ihn, Electronic Quantum Transport in Mesoscopic Semiconductor Structures (Springer-Verlag, New York, Inc., 2004).

[2] G. A. Prinz, Science 282, 1660 (1998).

[3] S. Saikin, J. Phys.: Condens. Matter 16, 5071 (2004).

[4] R. Merkt, M. Janssen, and B. Huckestein, Phys. Rev. B 58, 4394 (1998).

[5] Y. Li and C. R. Chang, J. Magn. Magn. Mater. 277, 344 (2004).

[6] T. P. Pareek and P. Bruno, Pramana-J. Phys. 58, 293 (2002).

[7] V. K. Dugaev, P. Bruno, and J. Barnaś, Phys. Rev. B 64, 144423 (2001).

[8] V. K. Dugaev, P. Bruno, and J. Barnaś, J. Magn. Magn. Mater. 242, 461 (2002).

[9] E. G. Miszczenko and B. J. Halperin, Phys. Rev. B 68, 045317 (2003). 
[10] T. Dittrich, P. Hänggi, G.-L. Ingold, B. Kramer, G. Schön, and W. Zwerger, Quantum Transport and Dissipation (Wiley-VCH, Weinheim, 1998).

[11] C. M. Varma, Z. Nussinov, and Wim van Saarloos, Phys. Rep. 361, 267 (2002).

[12] B. J. Hickey, D. Greig, and M. A. Howson, J. Phys. F 16, L13 (1986).

[13] A. Sahnoune, J. O. Ström-Olsen, and H. E. Fischer, Phys. Rev. B 46, 10035 (1992).

[14] F. M. Mayeya and M. A. Howson, J. Phys. C 4, 9355 (1992).

[15] P. Pratumpong, R. F. Cochrane, S. D. Evans, S. Johnson, and M. A. Howson, J. Phys.: Condens. Matter 14 11779 (2002).

[16] H. Fukuyama and K. Hoshino, J. Phys. Soc. Jpn. 50, 2131 (1981).

[17] A. Kawabata, Solid State Commun. 34, 431 (1980).

[18] A. Kawabata, J. Phys. Soc. Jpn. 49, 628 (1980).

[19] G. J. Morgan, A. Paja, and B. Spisak, J. Non-Cryst. Solids 270, 269 (2000).

[20] B. L. Altshuler and A. G. Aronov, in: Electron-Electron Interaction in Disordered Systems, edited by A. L. Efros and M. Pollak (North-Holland, Amsterdam, 1985).

[21] L. Liu, Phys. Rev. 126, 1317 (1962).

[22] L. Liu, Phys. Rev. Lett. 6, 683 (1961).

[23] I. Bialynicki-Birula, M. Cieplak, and J. Kaminski, Theory of Quanta (Oxford University Press, New York, 1992).

[24] L. D. Landau and E. M. Lifshitz, Quantum Mechanics (PWN, Warszawa, 1979).

[25] G. Bergmann, Phys. Rep. 107, 1 (1984).

[26] G. J. Morgan, M. A. Howson, and K. Saub, J. Phys. F 15, 2157 (1985).

[27] A. Pasturel and G. J. Morgan, J. Phys. F 17, 1561 (1987).

[28] A. Paja and G. J. Morgan, phys. stat. sol. (b) 206, 701 (1998).

[29] J. M. Ziman, Philos. Mag. 6, 1013 (1961).

[30] S. Hikami, A. I. Larkin, and Y. Nagaoka, Prog. Theor. Phys. Lett. 63, 707 (1980).

[31] R. Kubo, J. Phys. Soc. Jpn. 19, 2127 (1964).

[32] E. Wigner, Phys. Rev. 40, 749 (1932).

[33] S. Fujita, Introduction to Non-Equilibrium Quantum Statistical Mechanics (Saunders, London, 1966).

[34] J. M. Luttinger and W. Kohn, Phys. Rev. 108, 590 (1957).

[35] J. M. Luttinger and W. Kohn, Phys. Rev. 109, 1892 (1958).

[36] D. Vollhardt and P. Wölfle, Phys. Rev. B 22, 4666 (1980).

[37] J. S. Langer and T. Neal, Phys. Rev. Lett. 16, 984 (1966).

[38] P. Bordone, A. Bertoni, R. Brunetti, and C. Jacoboni, VLSI DESIGN 13, 211 Sp. Iss. SI (2001).

[39] K. Y. Kim and B. Lee, J. Appl. Phys. 86, 5085 (1999).

[40] M. Nedjalkov, I. Dimov, F. Rossi, and C. Jacoboni, Math. Comput. Model. 23, 159 (1996).

[41] K. Y. Kim and B. Lee, J. Appl. Phys. 85, 7252 (1999).

[42] C. Jacoboni, A. Bertoni, P. Bordone, and R. Brunetti, Math. Comput. Simul. 55, 67 (2001).

[43] M. B. Unlu, B. Rosen, H. L. Cui, and P. J. Zhao, Phys. Lett. A 327, 230 (2004).

[44] R. Zwanzig, Phys. Rev. 124, 983 (1961).

[45] H. Mori, Prog. Theor. Phys. 34, 399 (1965).

[46] N. A. Bruce and G. J. Morgan, Phys. Rev. B 51, 12313 (1995).

[47] G. J. Morgan and M. A. Howson, J. Phys. C 18, 4327 (1985).

[48] J. Smit, Physica (Amsterdam) 24, 39 (1958).

[49] B. Velicky, S. Kirkpatrick, and H. Ehrenreich, Phys. Rev. 175, 747 (1968).

[50] J. S. Langer, Phys. Rev. 120, 714 (1960).

[51] H. R. Brown and G. J. Morgan, J. Phys. F 1, 132 (1971).

[52] M. A. Howson and B. L. Gallagher, Phys. Rep. 170, 265 (1988).

[53] M. A. Howson, B. J. Hickey, and G. J. Morgan, Phys. Rev. B 38, 5267 (1988).

[54] G. Bergmann, Solid State Commun. 42, 815, (1982).

[55] P. A. Lee and T. V. Ramakrishnan, Rev. Mod. Phys. 57, 287 (1985).

[56] B. L. Altshuler, D. Khmel'nitzkii, A. I. Larkin, and P. A. Lee, Phys. Rev. B 22, 5142 (1980).

[57] S. Chakravarty and A. Schmid, Phys. Rep. 140, 193 (1986). 